\title{
Pore-scale Visualization of the Mobilization of a Partially Wetting 1 Droplet
}

\author{
Shao-Yiu Hsu ${ }^{\mathrm{a}, *}$, Markus Hilpert ${ }^{\mathrm{b}} 3$ \\ ${ }^{a}$ Graduate Institute of Hydrological and Oceanic Science, National Central University, No. 300, Jhongda 4 \\ Rd., Jhongli City, Taoyuan County, 320, Taiwan (ROC) 5 \\ ${ }^{b}$ Environmental Health Sciences, Johns Hopkins University, 615 N. Wolfe Street, Baltimore, Maryland, 6 \\ 21205, US
}

\begin{abstract}
We experimentally investigated the mobilization process of a partially wetting droplet in a pore doublet model. The process, involving contact line movement, liquid film generation, and interface deformation, has not been fully understood and modeled. In equilibrium, the droplet sat in one of the capillary tubes of the pore doublet, and the contact lines of the droplet were pinned with nonzero contact angles. The droplet was subject to pressure differences that were generated by pumping the continuous liquid into the pore doublet at different flow rates. At low flow rates, the droplet moved with sliding contact lines, and the shapes of the droplet's menisci roughly resembled spherical caps. When the flow rate increased, the velocity of the droplet increased, and the shapes of the liquid-liquid interfaces changed dramatically forming finger-like structures and films. Once films form, the classical Young-Laplace equation, which assumes a spherical interface, is not sufficient to describe droplet mobilization and the corresponding pressure drops. The films ruptured as the droplet moved forward, and a certain amount of droplet liquid was left behind. When the flow rates reached a certain level, the droplet snapped off from the tube wall and rolled while being surrounded by thin films formed by the continuous liquid.
\end{abstract}

\footnotetext{
${ }^{*}$ Corresponding author

Email address: syhsu@ncu.edu.tw (Shao-Yiu Hsu)
} 


\section{Introduction}

A good conceptual and quantitative understanding of the dynamic behavior of liquid- 10 liquid interfaces and trapped droplets in capillary tubes forms the basis for accurate predic- 11 tion of the mobilization of residual droplets during oil recovery, groundwater remediation, 12 and geological carbon sequestration (GCS) in geological porous media. In the past decades, 13 the flows of single interfaces and non-wetting phase droplets in capillary tubes have been 14 investigated [1, 2, 3, 4, 5, 6, 7, 8, 9]. However, the wettability of oil in petroleum reservoirs 15 varies, and contact angle hysteresis, a phenomenon closely related to partial wetting, cannot 16 be neglected [10, 11]. It turns out that our understanding of the mobilization of partially 17 wetting droplets is still very limited [12, 13].

On a solid surface (e.g., the wall of a capillary tube), the behaviors of completely wetting, 19 partially wetting, and non-wetting droplets are very different from each other. For a small 20 liquid droplet on a solid surface, the spreading coefficient, $S=\gamma_{S V}-\left(\gamma_{S L}+\gamma_{L V}\right)$, defines the ${ }^{21}$ equilibrium shape of the droplets, where $\gamma_{L V}, \gamma_{S V}$, and $\gamma_{S L}$ are the liquid-gas, solid-gas, and 22 solid-liquid interfacial tensions, respectively [14]. For $S>0$, a spreading system takes shape ${ }^{23}$ or total or complete wetting occurs: a droplet spreads and tends to cover the solid surface 24 forming a thin film on the solid. For $S<0$, a non-spreading or partially wetting system 25 occurs: a three-phase contact line is formed, and a non-zero contact angle between the fluids ${ }_{26}$ and the solid surface is established. In addition, on a rough surface, the wettability can be ${ }_{27}$ manipulated directly by the roughness of the surface [15, 16, 17]. 28

A non-wetting droplet(e.g. an air bubble in a capillary tube consisting of glass and oth- 29 erwise filled with water) is often completely surrounded by the wetting liquid that forms a 30 very thin film between the droplet and the tube wall as shown in Figure 1(1). Once the 31 non-wetting droplet is mobilized, the thickness of the film depends on the droplet velocity 32 [1, 18. In a partially wetting situation, however, the liquid-liquid interfaces exhibit non-zero 33 contact angles with the tube wall as shown in Figure 11(2) [13]. 34

In straight capillary tubes, the mobilities of partially wetting droplets and non-wetting 35 droplets differ significantly. A non-wetting droplet can move freely while a partially wetting 36 
droplet can be trapped owing to contact angle hysteresis and can be mobilized only when 37 the external pressure difference overcomes the capillary pressures [19, 11]. 38

Studies have shown that the mobilization of a droplet in the partially wetting regime 39 involves contact line movement and liquid film generation [12, 13]. Either the contact lines 40 of a partially wetting droplet can move in a sliding motion while maintaining contact with the ${ }_{41}^{41}$ solid surface, or the meniscus can move over a thin continuous liquid film while assuming 42 a finger-like shape [18, 20, 21, 22, 23, 24, 14, 25, 26, 27, 13]. The occurrence of sliding 43 contact lines and finger-like shape depends on the droplet velocity or the capillary number, 44 $C a=U \eta_{c} / \gamma$, where $U$ is the velocity of the liquid finger tip and $\eta$ is the viscosity of the ${ }_{45}$ surrounding liquid. In addition, the shape of the finger and the thickness of the liquid film 46 should be related to capillary number [18, 25, 13].

Dong et al. [12 experimentally showed that a partially wetting droplet was moved when 48 the surrounding liquid formed a thin film at the advancing meniscus as the droplet started to 49 move away from its original location. Eventually, the droplet was completely surrounded by 50 the film, i.e., a transition of the droplet from initially partial wetting to non-wetting occurred. 51 On the other hand, a partially wetting droplet was also mobilized with contact lines sliding 52 or slipping on a solid surface [20, 27, 11, 28]. No thin surrounding liquid films were formed. ${ }_{53}$ In addition, Luo et al. [29] and Oskooei and Sinton [27] showed that the roughness and 54 wettability of tube surfaces could affect both the contact line movement and film generation. $\quad 55$ The circumstances of film generation and transient behaviors during droplet mobilization 56 have not been systematically investigated.

In this paper, we present visualization experiments of droplet mobilization in a pore ${ }_{58}$ doublet in the partially wetting regime. We show that the mechanisms that result in 59 droplet movement are highly dependent on the magnitude of the external pressure differ- 60 ences and droplet velocities. 


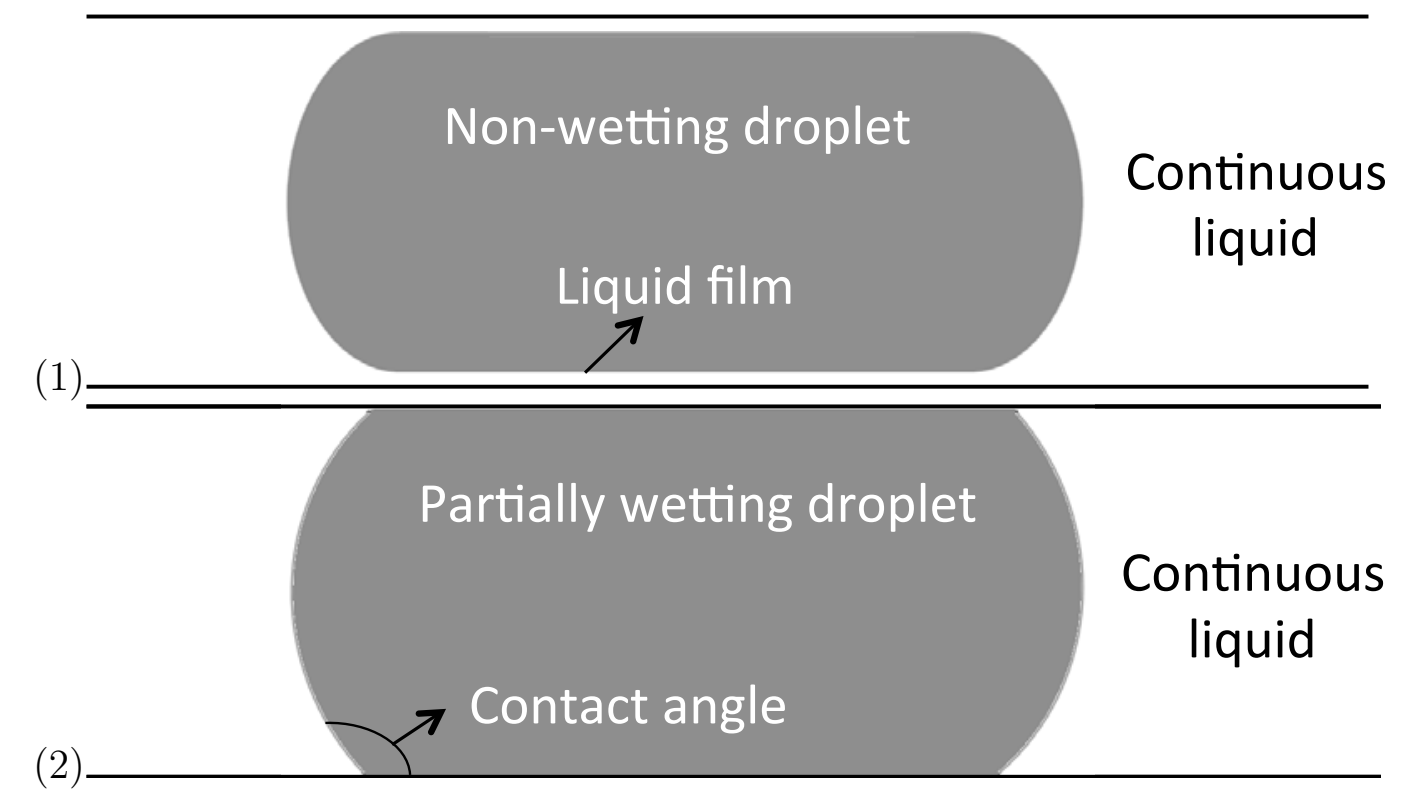

Figure 1: Schematics of (1) a non-wetting droplet and (2) partially wetting droplet in equilibrium states

\section{Methods}

\subsection{Pore doublet}

We performed droplet mobilization experiments within a pore doublet, a device previ- ${ }_{64}$ ously used in studies of two-phase flow in porous media [30, 11]. A pore doublet is the 65 simplest model that enables one to mimic the mobilization of trapped droplets in topologi- ${ }_{66}$ cally complex porous media by means of flooding with another immiscible fluid.

The pore doublet comprises two straight capillary tubes, each with a circular cross- ${ }_{68}$ section (see Figure 2). Tube 1 is formed by a hole with a diameter of $2 \mathrm{~mm}$ that was drilled ${ }_{69}$ perpendicularly into one of an acrylic rectangular prism's faces. Tube 2 is a straight capillary 70 tube (PTFE) with an inside diameter of $1.9 \mathrm{~mm}$. A T-connector at point A connects an end 71 of each of the two tubes to a liquid reservoir; and another T-connector at point B connects 72 the other two ends of the tubes to another T-connector, which receives flow from a syringe 73 pump and an actuator. The total length of each tube between points A and B is $242 \mathrm{~mm}$. 74

The syringe pump $\left(500 \mathrm{D}\right.$, Teledyne $\left.\mathrm{ISCO}^{T M}\right)$ can provide a very steady flow $\left(0.5 \%\right.$ flow ${ }_{75}$ accuracy) at rates ranging from 0.001 to $204 \mathrm{ml} / \mathrm{min}$. A pressure difference between point 76 $\mathrm{A}$ and point $\mathrm{B}$ can be generated by pumping a continuous liquid into the pore doublet at a 77 
constant flow rate. We refer to this flow as "steady background flow." We used two pressure 78 transducers (PX 170, Omega) on both sides of the pore doublet to measure the pressure 79 drop. More details of the pore doublet are given in Hsu et al. [11]. 80

To test the macro-scale homogeneity of the surface of Tube 1, we applied a constant 81 and small pressure difference across the capillary tube, and the droplet was mobilized and 82 moved in a constant and very slow pace. We found that the dynamic advancing and receding 83 contact angles of the moving droplet did not change during the sweeping process. Therefore, 84 the roughness and wettability of the tube surface could be assumed to be uniform at the 85 macro scale.

\subsection{Droplet visualization}

The planar laser-induced fluorescence (PLIF) and refractive index matching (RIM) tech- $\quad 88$ niques, non-invasive methods for flow visualization [31, 11], enabled us to visualize droplet dy- 89 namics in Tube 1 of the pore doublet. We chose two immiscible liquids (Code 5095 and OHZB 90 from Cargille Lab), both of which possess the same refractive index as acrylic (i.e., 1.49). 91 The properties of the two liquids are shown in Table $\mathbf{1}$.

To visualize the blob, we added the fluorescent dye Sulforhodamine $G$ (absorption wave- ${ }_{93}$ length $532 \mathrm{~nm}$, emission wavelength $548 \mathrm{~nm}$ ) to the liquid $O H Z B$, which was injected into 94 the Code 5095-filled Tube 1 for the formation of a droplet. Note that the interfacial tension 95

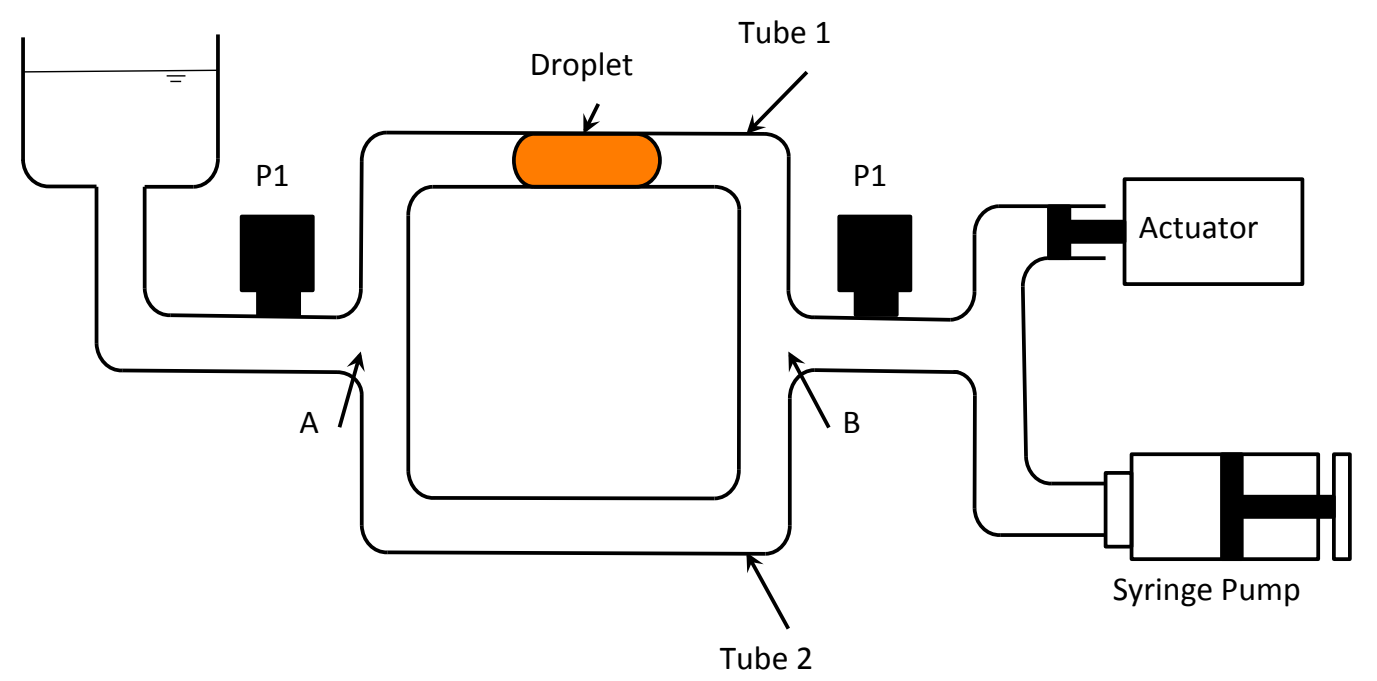

Figure 2: Sketch of our experimental pore doublet model. P1 and P2 are pressure transducers. 
Table 1: Properties of liquid phases at $25^{\circ} \mathrm{C}$.

\begin{tabular}{lcc}
\hline & $\begin{array}{c}\text { Continuous liquid } \\
\text { Code 5095 }\end{array}$ & $\begin{array}{c}\text { Droplet liquid } \\
\text { OHZB }\end{array}$ \\
\hline Density $\rho\left(\mathrm{g} / \mathrm{cm}^{3}\right)$ & 0.87 & 2.06 \\
Dynamic viscosity $\eta(\mathrm{mPa} \cdot \mathrm{s})$ & 13 & 10.3 \\
Interfacial tension $\sigma(\mathrm{dyne} / \mathrm{cm})$ & \multicolumn{2}{c}{6.72} \\
\hline
\end{tabular}

shown in Table 1 was measured between the continuous liquid and the dyed droplet liq- 96 uid. Code 5095 is called the "continuous liquid," and OHZB is called the "droplet liquid." 97 The droplet was illuminated by a laser light sheet (wavelength $532 \mathrm{~nm}$ ) and recorded by a 98 PC-controlled high-speed CMOS digital camera (Basler 504Kc). The images were processed 99 to detect phase boundaries with image analysis software tools. The 2-mm tube diame- ${ }_{100}$ ter enabled us to discretize vertically a cross-sectional image of a droplet by 140 pixels (14 101 $\mu \mathrm{m} /$ pixel) for a clear observation of the interface dynamics and contact angles measurement, 102 but inevitably allowed gravity to affect the droplet shape. 103

\subsection{Experimental procedure and measurements}

104

We performed droplet mobilization experiments with steady background flows for five ${ }_{105}$ different pumping rates that ranged from 0.2 to $0.8 \mathrm{ml} / \mathrm{min}$. For each pumping rate, the 106 following five major actions were taken: (1) the tubes and flow cell were cleaned with DI ${ }_{107}$ water and carefully dried; (2) after filling the entire pore doublet model with the continuous 108 liquid, we injected $10 \mu \mathrm{l}$ of the fluorescent liquid into Tube 1 to form a droplet; (3) we 109 illuminated the central cross-section of the droplet with a laser light sheet; (4) we tested ${ }_{110}$ whether the droplet was trapped in Tube 1 by pumping the continuous liquid into the pore ${ }_{111}$ doublet at a relatively small pumping rate of $0.1 \mathrm{ml} / \mathrm{min}$, and we proceeded to the next step 112 only if the droplet could then not be mobilized; and (5) we increased the pumping rate to 113 the target pumping rate $(\geq 0.2 \mathrm{ml} / \mathrm{min})$ and imaged droplet movement while also recording ${ }_{114}$ the pressures on both sides of the pore doublet. 


\section{Results}

\subsection{Equilibrium state}

Once a droplet was injected into Tube 1, we applied no flow $\left(Q_{t}=0.0 \mathrm{ml} / \mathrm{min}\right)$ and waited ${ }_{118}$ a certain period of time so that the droplet could assume an equilibrium state, in which 119 contact angles would remain constant. The liquid-liquid interfaces of the droplets formed 120 well-defined contact angles, as illustrated in Figure 3 . Note that, owing to the density ${ }_{121}$ difference between liquids, the contact angle was not uniform along a contact line [32, 33, 11]. 122 The contact angle values that we present in this manuscript are average values of the apparent ${ }_{123}$ contact angle measured on the top and at the bottom in the cross-sectional images of the ${ }_{124}$ droplets. For the droplet shown in Figure 3, the measured apparent equilibrium contact 125 angle amounted to $75^{\circ}$. The droplet was in the partial wetting regime, and the two liquids 126 were in a state of near-neutral wettability. 127

\subsection{Steady background flows}

Experiments were performed for five different pumping rates where $Q_{t} \geq 0.2 \mathrm{ml} / \mathrm{min}$. The ${ }_{129}$ droplet behavior depended significantly on $Q_{t}$. On the basis of the contact line movement 130 and interface deformation, we identified the following four different flow regimes: (1) sliding, 131 (2) sliding and fingering, (3) fingering-induced droplet collapse, and (4) fingering-induced 132 droplet rolling. Experimental results for these flow regimes are presented in the following 133 sections.

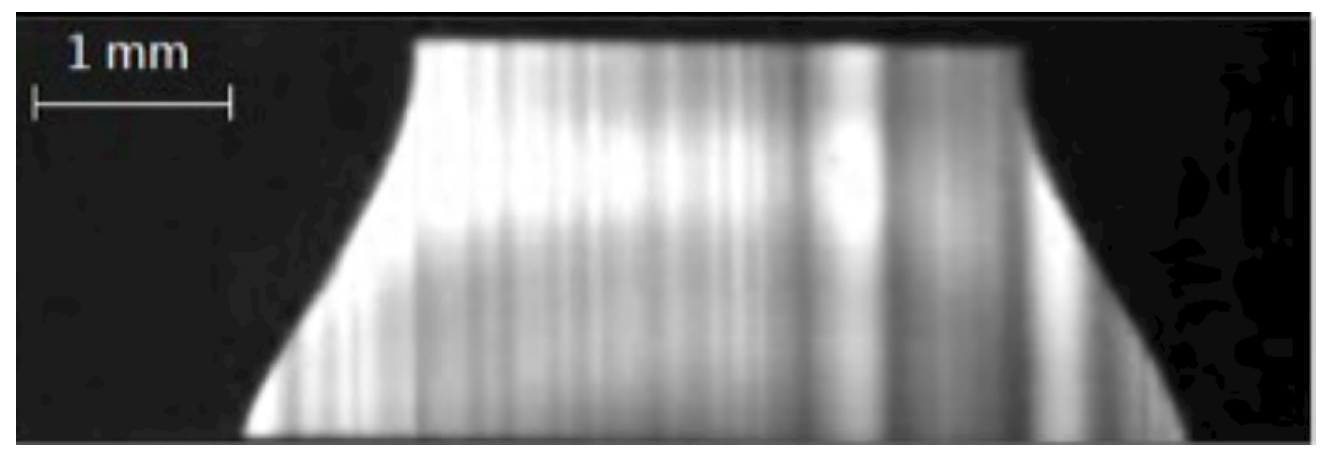

Figure 3: Snapshot of a droplet in an equilibrium state in the pore doublet. 


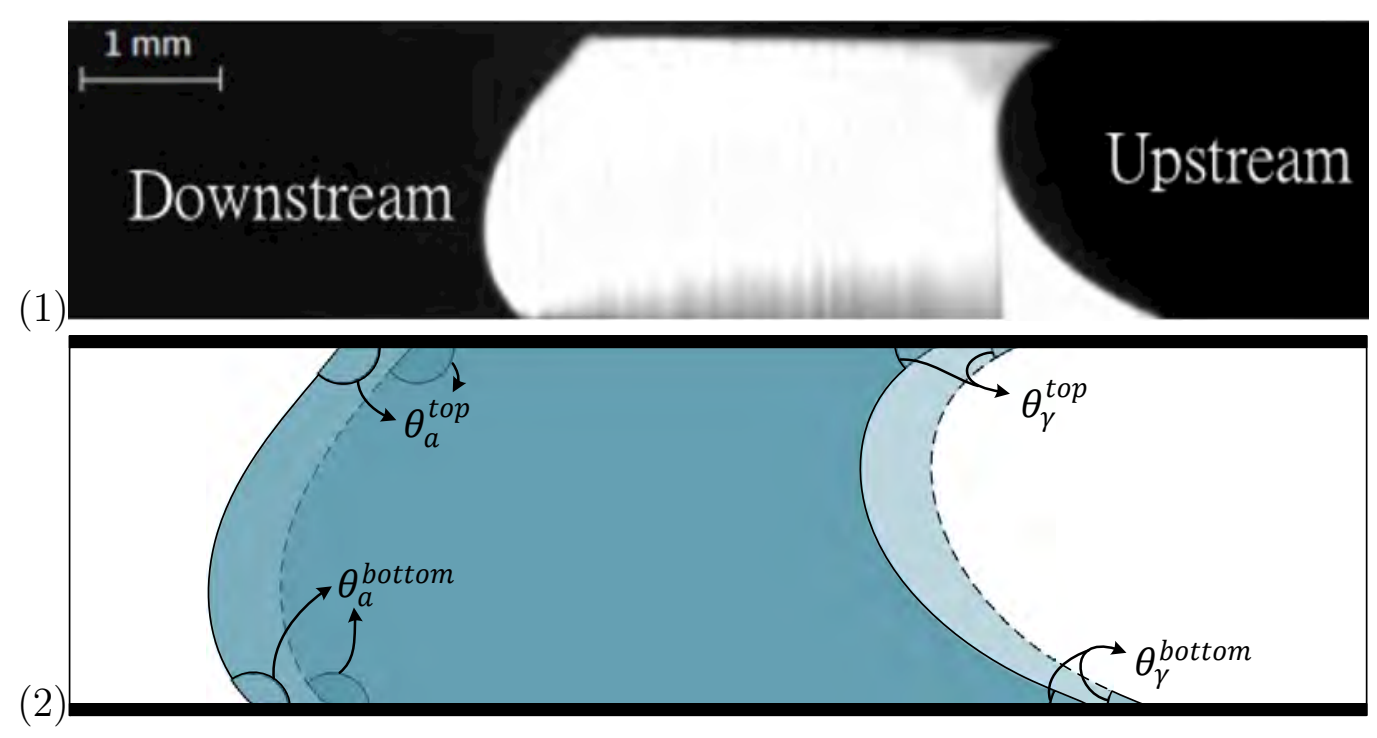

Figure 4: (1) Snapshot of a mobilized droplet for a pumping rate $Q_{t}=0.2 \mathrm{ml} / \mathrm{min}$. (2) Schematic of the droplet moving in Flow Regime I. The dashed line is the shape and position of the droplet at $\mathrm{t}=60 \mathrm{~s}$, and the solid line is the droplet at $\mathrm{t}=140 \mathrm{~s}$. $\theta_{a}^{\text {top }}$ and $\theta_{r}^{\text {top }}$ are the advancing and receding contact angles along the top of the tube wall. $\theta_{a}^{\text {bottom }}$ and $\theta_{r}^{\text {bottom }}$ are the advancing and receding contact angles along the bottom of the tube wall.

\subsubsection{Flow Regime I: Sliding $\left(Q_{t}=0.2\right.$ and $\left.0.3 \mathrm{ml} / \mathrm{min}\right)$}

In this flow regime, the droplet moved at a very slow pace with liquid-liquid interfaces 136 "sliding" over the solid surface at the two three-phase contact lines. On the micro scale, 137 the moving interfaces might undergo an unsteady "stick-slip" motion [34, 7, 29]. However, 138 since the "stick" motion could not directly be observed in our experiments, we refer to 139 the movement of the interface as "sliding". Neither did we observe any thin liquid film 140 between the droplet and the tube wall at the downstream meniscus of the droplet, nor any ${ }_{141}$ droplet liquid that was left between the continuous liquid and the tube wall behind the ${ }_{142}$ upstream meniscus of the droplet.

For $Q_{t}=0.2 \mathrm{ml} / \mathrm{min}$, the droplet was mobilized when the contact angles slightly trans- ${ }_{144}$ gressed the "hysteresis region," which was bounded by the equilibrium advancing and reced- ${ }_{145}$ ing contact angles [35, 36]. Figure 4(1) shows a snapshot of a droplet moving from upstream 146 to downstream (from right to left). Figure 4(2) is a schematic figure of a droplet moving ${ }_{147}$ with sliding contact lines and constant contact angles in Flow Regime I. 
In this flow regime, a finger that leaves a film of the surrounding liquid in a capillary tube ${ }_{150}$ was formed by the continuous liquid at the upstream liquid-liquid interfaces, and thin films 151 consisting of droplet liquid were generated behind the upstream interfaces at both the top and 152 the bottom of the tube, as illustrated in Figure 5(1). Once the thin films were formed, the ${ }_{153}$ liquid-liquid interfaces were changed to a finger-like shape [37, 24, 38, 39, 12, 13]. Moreover, 154 as shown in Figure 5(2)(3), the droplet liquid films ruptured during the mobilization 155 process, and small portions of droplet liquid were left behind the main moving droplet on the 156 top and bottom of the tube wall. Actually, the top film was not detectable in Figure $\mathbf{5}(\mathbf{1}), \quad 157$ but a small droplet on the top of the tube behind the main droplet serves as evidence of ${ }_{158}$ the existence of the top droplet liquid film and its rupture. Note that the image contrast 159 was enhanced locally around the small droplet. After the rupture, the main droplet kept 160 moving while maintaining the finger and sliding contact lines. We refer to this flow regime as ${ }_{161}$ "sliding and fingering."

\subsubsection{Flow Regime III: Fingering-induced droplet collapse $\left(Q_{t}=0.6 \mathrm{ml} / \mathrm{min}\right){ }^{163}$}

At the beginning of the droplet mobilization in this flow regime, both downstream and 164 upstream fingers and films were formed, as shown in Figure 6(1). Figure 6(2) shows the 165 blob at time $\mathrm{t}=15.34 \mathrm{~s}$, the critical state, in which the top part of the upstream finger ${ }_{166}$ was approaching the continuous liquid film on the top of the downstream finger, and shortly ${ }_{167}$ afterward, the top part of the moving droplet detached from the tube wall. At time $t=15.46 \quad 168$ s, the droplet collapsed, as shown in Figure 6(3). At this stage, the continuous liquid 169 passed over the droplet, and the collapsed droplet became immobile. Figure 7 shows the 170 displacement of the tip of the downstream interface of the droplet and for $Q_{t}=0.6 \mathrm{ml} / \mathrm{min}, \quad{ }^{171}$ the evolution of the pressure drop across the pore doublet model, and the snapshots of the 172 droplet at the moments before and after the droplet collapse. Because the droplet collapse ${ }_{173}$ was due to the liquid films and the fingers, we refer to this flow regime as "fingering-induced 174 droplet collapse". 
(1)

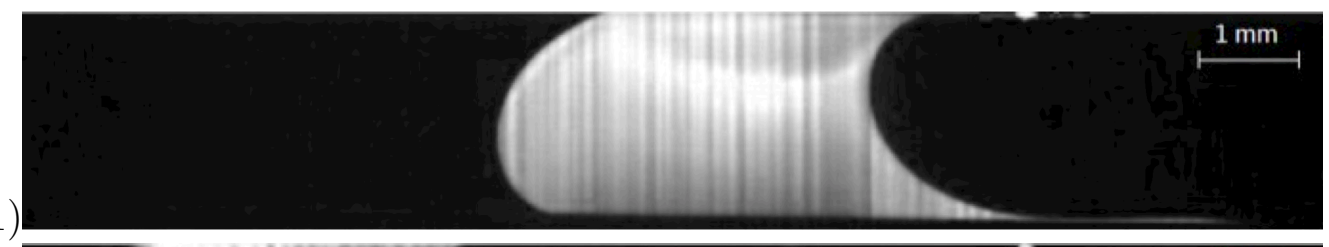

(2)
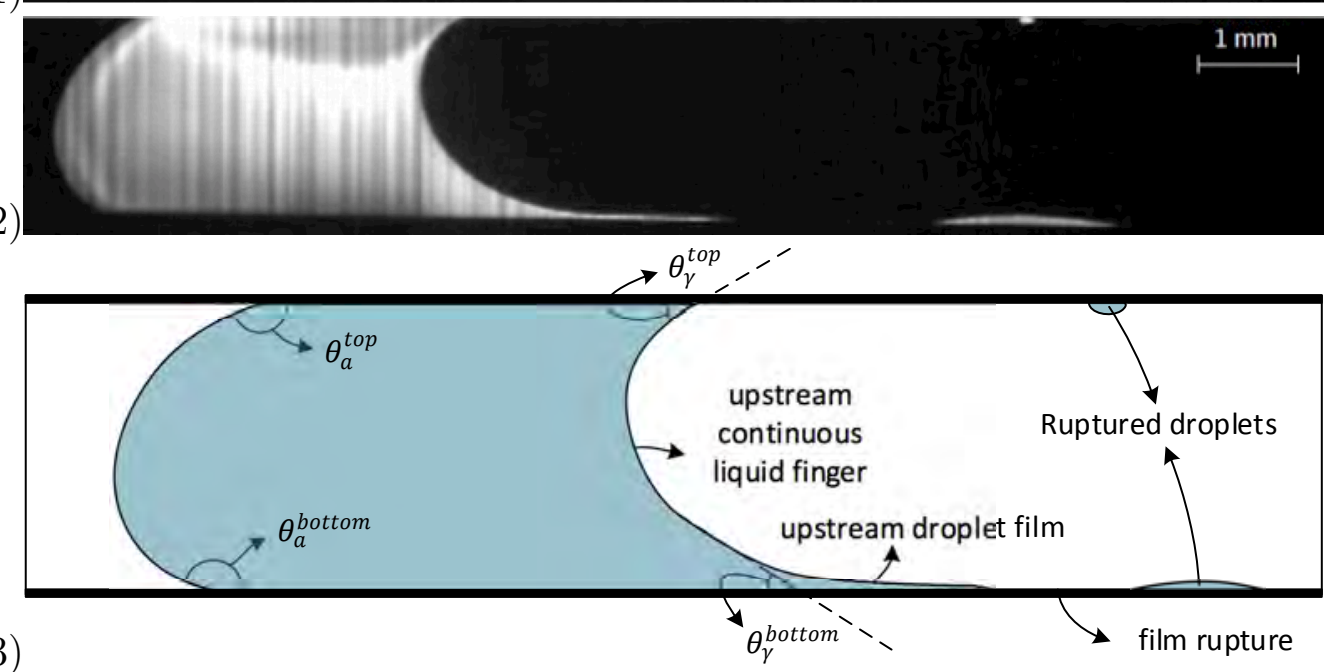

(3)

Figure 5: Snapshots of mobilized droplets for a pumping rate $Q_{t}=0.4 \mathrm{ml} / \mathrm{min}$ at (1) time $\mathrm{t}=23.1 \mathrm{~s}$ when the top film ruptured, and (2) $\mathrm{t}=45.0 \mathrm{~s}$ when the bottom film ruptured. (3) Schematic of liquid fingers and films and film rupture in (2)

$(1)$

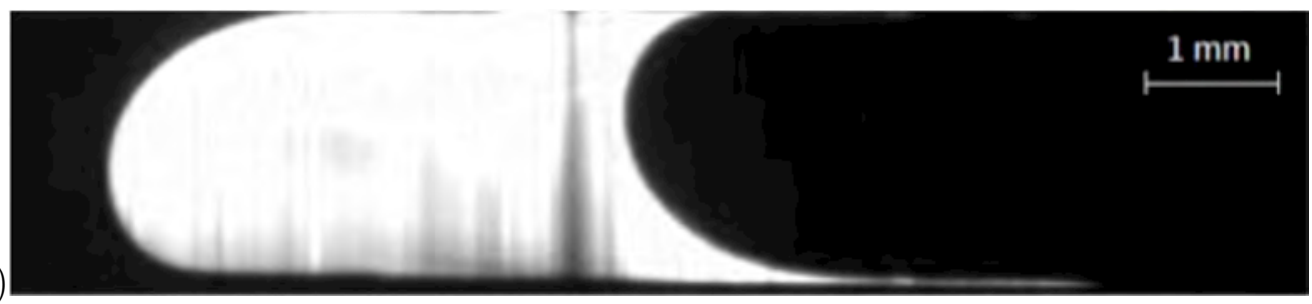

$(2)$
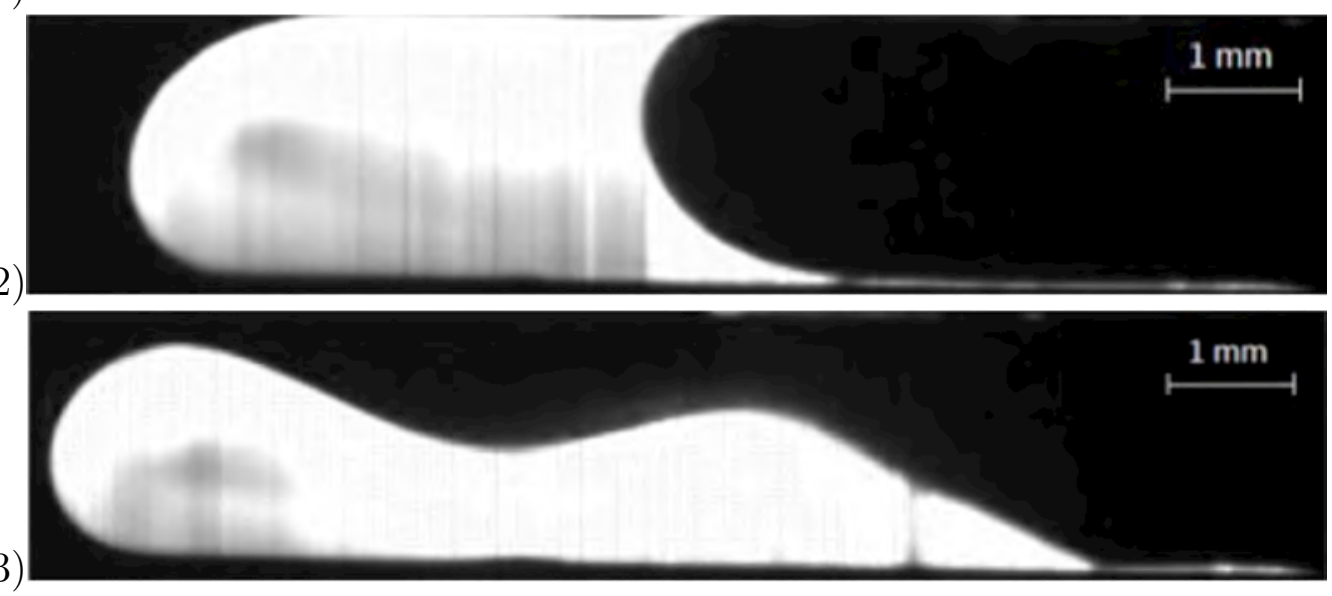

Figure 6: Snapshots of mobilized droplets for a pumping rate $Q_{t}=0.6 \mathrm{ml} / \mathrm{min}$ at $(1) t=10.00 \mathrm{~s}$ when the droplet moved with fingers and films, (2) $t=15.34 \mathrm{~s}$ when the droplet was at the critical moment before collapsing, and (3) $t=15.46 \mathrm{~s}$ when the droplet collapsed. 


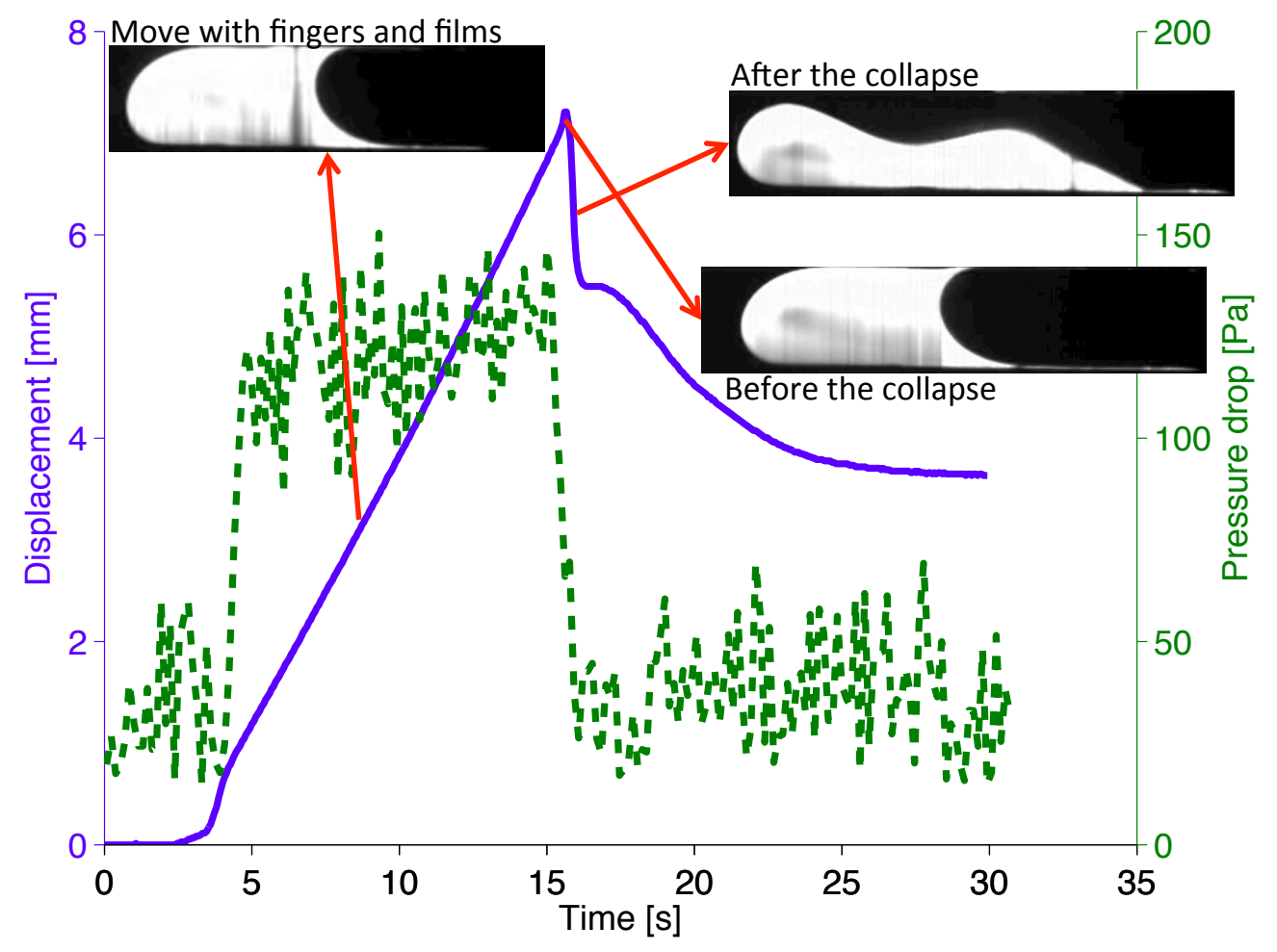

Figure 7: The displacement of the tip of the downstream liquid-liquid interface of a mobilized droplet (blue solid line) and the pressure drop across the pore doublet model (green dashed line) versus time for a pumping rate $Q_{t}=0.6 \mathrm{ml} / \mathrm{min}$.

The sub-images are snapshots of the droplet moving with fingers and films, before the collapse, and after the collapse. 


\subsubsection{Flow Regime IV: Fingering-induced droplet rolling $\left(Q_{t}=0.8 \mathrm{ml} / \mathrm{min}\right)$}

Just as with the moving droplet in the "fingering-induced collapse" flow regime, two 177 fingers were generated at the beginning of the mobilization process, as shown in Figure 8(1). 178 At $t=12.10 \mathrm{~s}$, as shown in Figure 8(2), the upstream finger came very close to the ${ }_{179}$ continuous liquid film, and a very thin droplet liquid film connected the droplet to the 180 bottom of the tube wall. Immediately afterward, at $t=14.00 \mathrm{~s}$, the thin film snapped off ${ }_{181}$ from the tube wall. Instead of the droplet collapse reported for the previous flow regime, 182 the droplet rolled forward, as shown in Figure 8(3). Figure 9 shows the displacement of ${ }_{183}$ the tip of the downstream interface of the droplet for $Q_{t}=0.8 \mathrm{ml} / \mathrm{min}$, the evolution of ${ }_{184}$ the pressure drop across the pore doublet model, and the snapshots of the droplet at the ${ }_{185}$ moments before and after the droplet's rolling. Because the rolling of droplet was due to the ${ }_{186}$ presence of two fingers, we named this flow regime "finger-induced rolling." At this stage, the ${ }_{187}$ droplet moved into the non-wetting regime where the contact angles between droplet liquid 188 and the solid surface on the two sides of the droplet were close to $180^{\circ}$. ${ }^{189}$

\subsubsection{Contact angle and droplet velocity}

As shown in Figure 10, we measured the dynamic advancing and receding contact angles, 191 $\theta_{a}$ and $\theta_{r}$, and the average moving velocities of the droplet subject to different pumping 192 rates. The advancing contact angle was measured on the downstream liquid-liquid interface ${ }_{193}$ where the droplet liquid displaced the continuous liquid, while the receding contact angle 194 was measured on the upstream liquid-liquid interface where the continuous liquid displaced 195 the droplet liquid. The contact angle values are average values of the apparent contact 196 angle measured on top, $\theta_{a / r}^{\text {top }}$, and at the bottom, $\theta_{a / r}^{b o t t o m}$, in the cross-sectional images of the ${ }_{197}$ droplets, as showed in Figure $4(2)$.

During the early stage of droplet mobilization in the flow regime of "fingering-induced 199 droplet rolling", the droplet velocity was $0.65 \mathrm{~mm} / \mathrm{s}$, and the advancing and receding contact 200 angles were $162^{\circ}$ and $22^{\circ}$, respectively. When the droplet started rolling, the velocity of the ${ }^{201}$ moving droplet changed to $1.22 \mathrm{~mm} / \mathrm{s}$. The advancing contact angle slightly decreased while 202 the receding contact angles dramatically changed from less than $90^{\circ}\left(22^{\circ}\right)$ to a value larger ${ }_{203}$ 
(1)

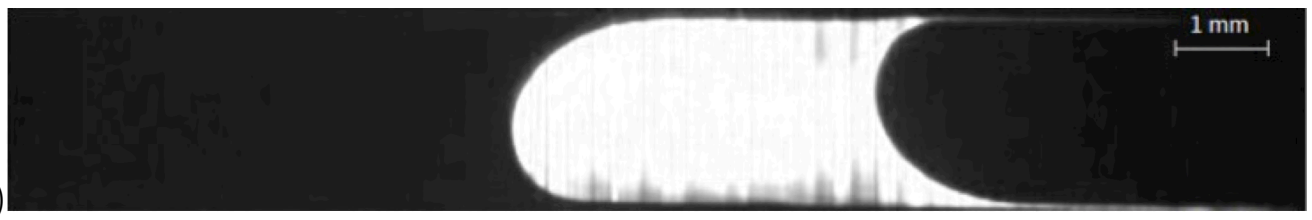

(2)

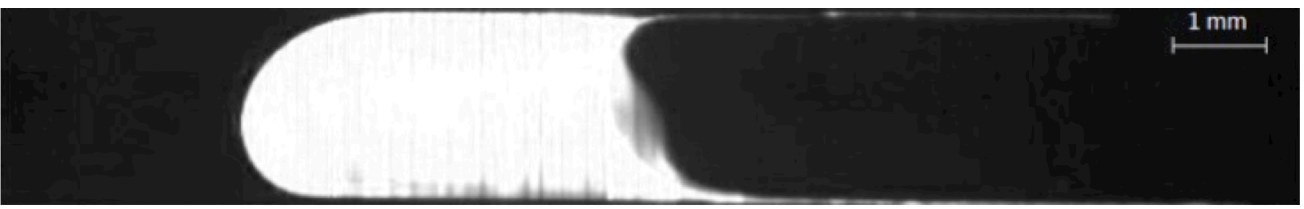

(3)
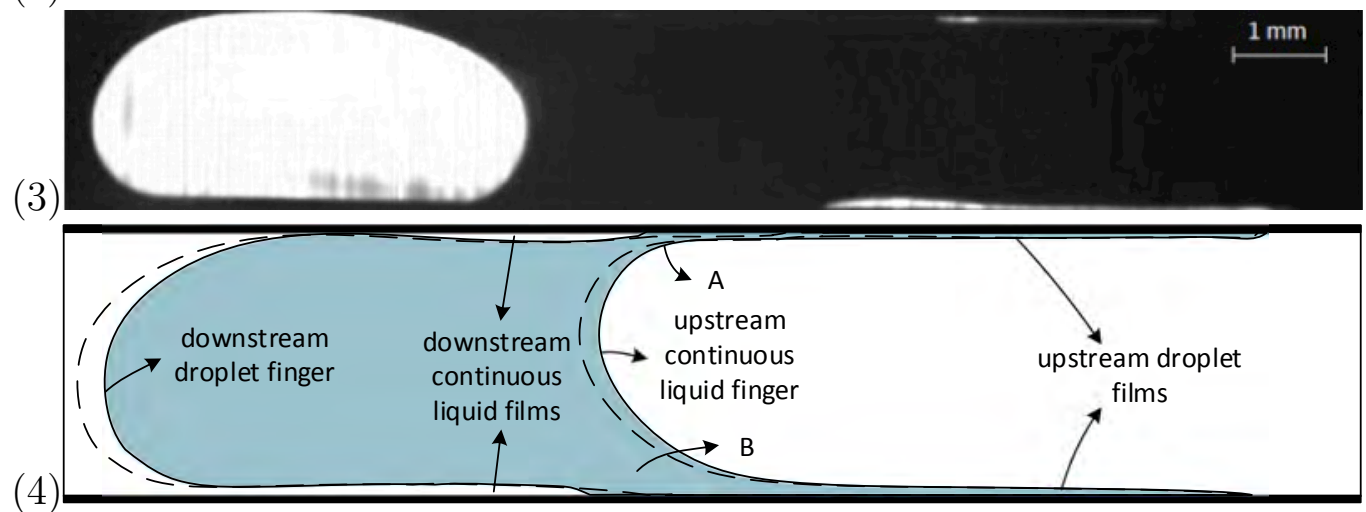

Figure 8: Snapshots of mobilized droplets for a pumping rate $Q_{t}=0.8 \mathrm{ml} / \mathrm{min}$ at times (1) $t=8.00 \mathrm{~s}$ when the droplet moved while forming fingers and films, (2) $t=12.10 \mathrm{~s}$, the critical moment just before rolling, and (3) $t=14.00 \mathrm{~s}$ after rolling. (4) Schematic of the droplet in the critical conditions: before collapse in Flow Regime III (solid line), and before rolling in Flow Regime IV (dashed line). Both of the upstream and downstream fingers and films were generated. 


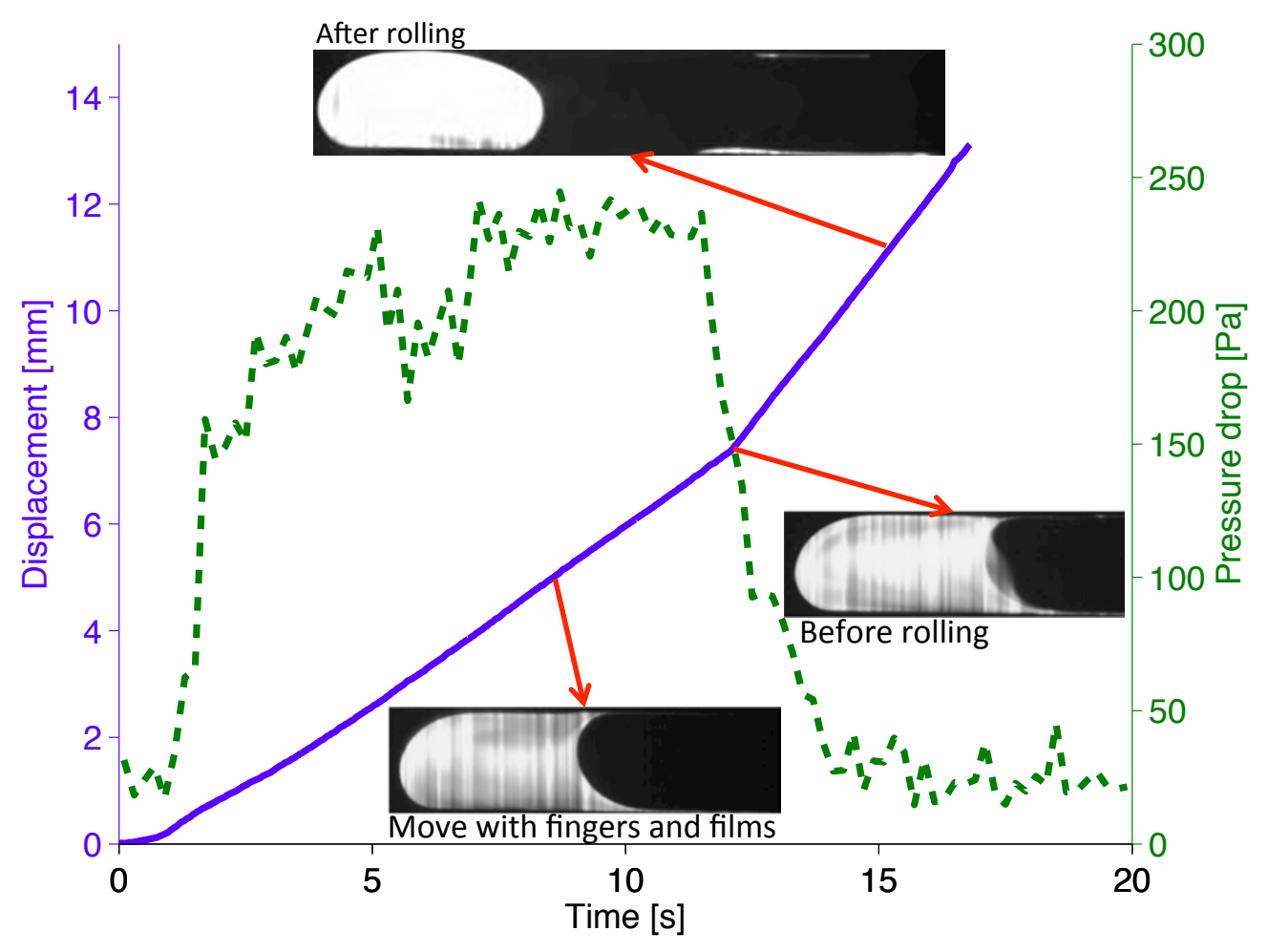

Figure 9: The displacement of the tip of the downstream liquid-liquid interface of a mobilized droplet (blue solid line) and the pressure drop across the pore doublet model (green dashed line) versus time for a pumping rate $Q_{t}=0.8 \mathrm{ml} / \mathrm{min}$. The sub-images are the snapshots of the droplet moving with fingers and films, before rolling, and after rolling. 


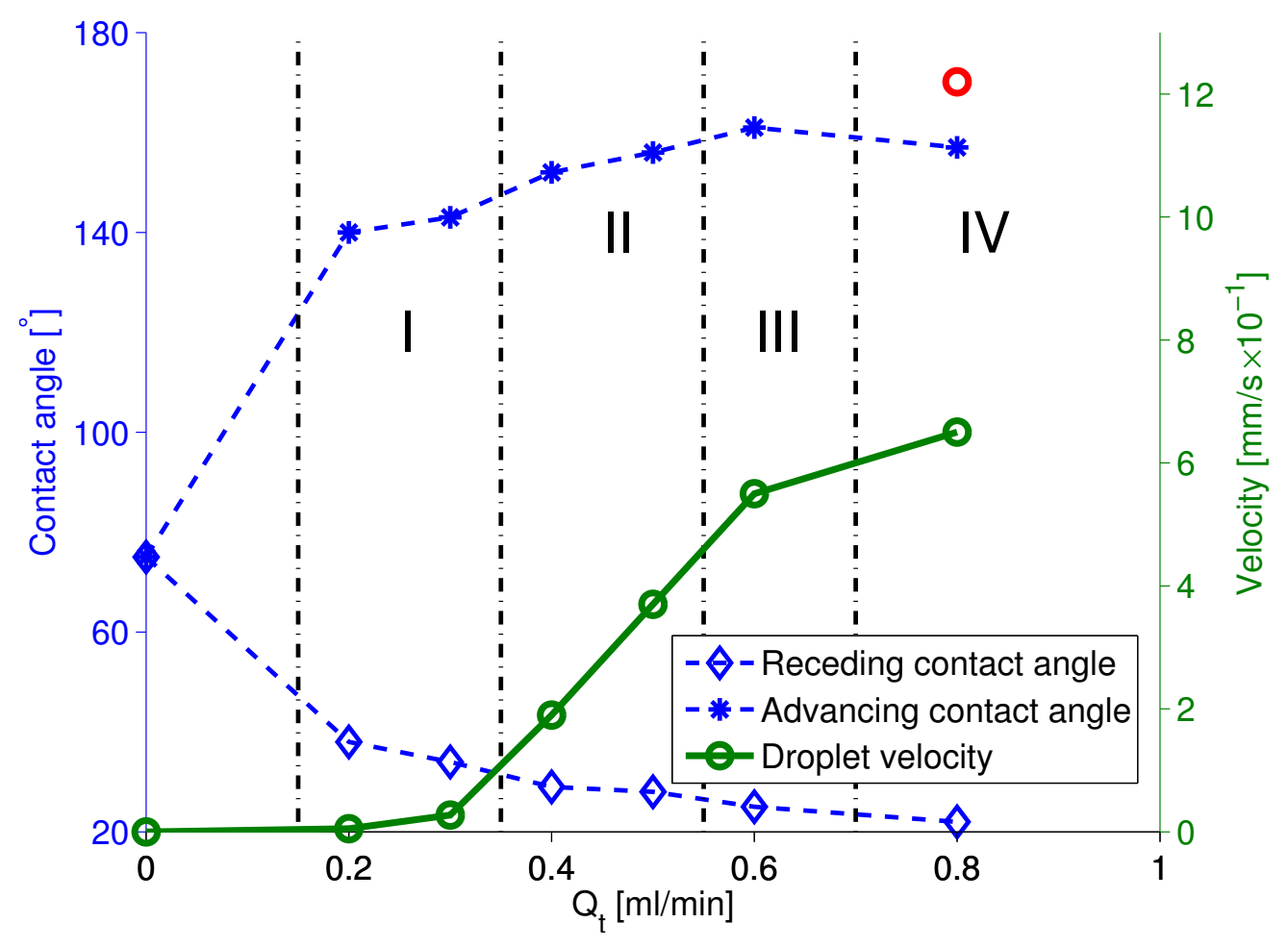

Figure 10: The measured contact angles, droplet moving velocities, and Flow Regimes for droplet mobilization due to steady background flows. The Flow Regimes are separated by dashed lines. In Flow Regimes IV, the green circle represents the droplet moving velocity before droplet rolling, while the red circle represents the droplet moving velocity after droplet rolling. 
than $90^{\circ}$. We suspect that a circular and thin continuous liquid film existed between tube ${ }_{204}$ wall and the droplet. 205

\section{Discussion 206}

\subsection{Flow Regimes of droplet mobilization versus capillary number ${ }_{207}$}

The processes that occur when a contact line of a partially wetting droplet moves on 208 a flat surface or in a capillary tube are still not entirely understood. The contact lines of ${ }_{209}$ a partially wetting droplet either can move in a sliding motion while maintaining contact ${ }_{210}$ with the solid surface or can move over a thin liquid film while assuming a finger-like shape ${ }_{211}$ $[18,20,21,22,23,24,14,26,27,13$. Our results show that the occurrence of sliding contact 212 lines and fingering depends on the droplet velocity or the capillary number, $C a=U \eta_{c} / \sigma,{ }^{213}$ where $U$ is the velocity of the liquid finger tip and $\eta_{c}$ is the viscosity of the continuous liquid. ${ }^{214}$ In addition, the shape of the fingers and thickness of the liquid films in Flow Regime II should ${ }_{215}$ also be related to capillary number [18, 13. Figure $\mathbf{1 1}$ shows the flow regime depending on ${ }_{216}$ $Q_{t}$ and the $\mathrm{Ca}$ of a moving droplet. Although no previous studies described finger-induced ${ }_{217}$ collapse and rolling in Flow Regimes III and IV, we expect the occurrence of the four different ${ }_{218}$ flow scenarios to be controlled by capillary number. ${ }_{219}$

It has also been shown that a change in surface properties affects not only interface 220 movement [40, 16, 41] but also - and therefore - multiphase flow in porous media at the ${ }_{221}$ Darcy scale [42]. Luo et al. [29] and Oskooei and Sinton [27] showed that, in capillary tubes, 222 increasing the roughness or changing the wettability of the tube surface will turn a droplet in ${ }^{223}$ Flow Regime I into a droplet in Flow Regime II or even Flow Regime IV, without a change ${ }_{224}$ in capillary number. Therefore, other nondimensional parameters that account, for example, ${ }^{225}$ for surface roughness can be expected to affect droplet mobilization and its dependence on 226 capillary number; however, our experiments did not resolve this additional dependence. 227

\subsection{Flow Regimes and dynamics of liquid-liquid interface ${ }_{228}$}

We experimentally demonstrated the existence of four different flow regimes for droplet mo- ${ }^{229}$ bilization in a pore doublet, to which a steady background flow was applied. In the following 230 sections, we tried to link the observed interface dynamics during droplet mobilization to ex- ${ }^{231}$ 


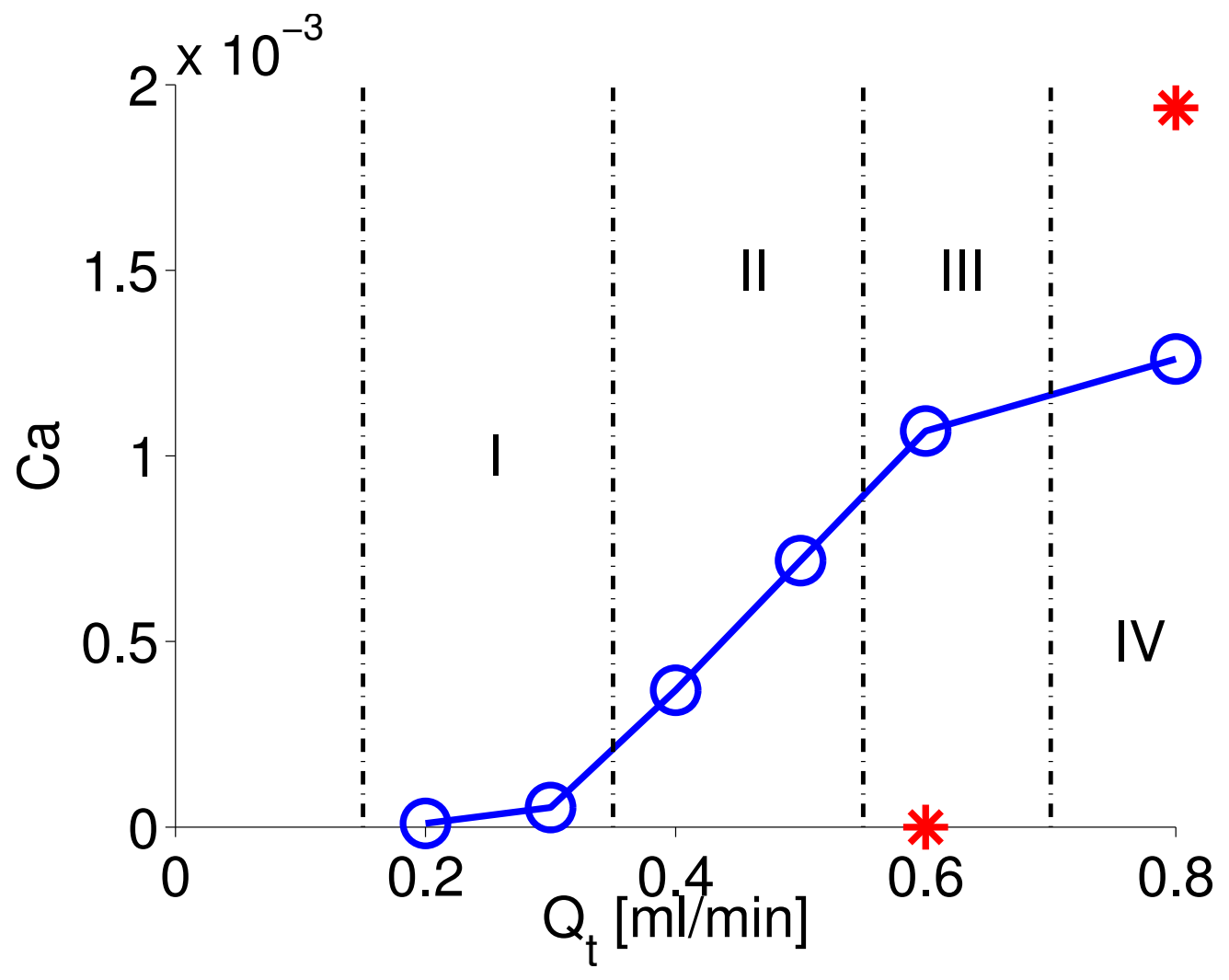

Figure 11: The capillary number and Flow Regimes for droplet mobilization due to steady background flows. The Flow Regimes are separated by dashed lines. In Flow Regimes III and IV, the circles represent the $C a$ values before droplet collapse and rolling, while the stars represent the $C a$-values after droplet collapse and rolling. 
isting two-phase flow theories and experimental observations documented in the literature. ${ }_{232}$

4.2.1. Sliding contact lines without fingers and films 233

In Flow Regime I, $C a \approx 10^{-5}$, where the capillary pressure dominated over the viscous ${ }^{234}$ stress, the droplet moved with three-phase contact lines sliding over the wall of the capillary ${ }_{235}$ tube. No liquid film was generated or deposited as shown in Figure 4. Nevertheless, 236 according to Taylor's law, the thickness of the continuous or droplet liquid film can, in ${ }_{237}$ general, be less than $10 \mu \mathrm{m}$ when $\mathrm{Ca}$ is around $10^{-5}$ [25]. Since the optical resolution of our ${ }^{238}$ system is limited to $14 \mathrm{um}$ per pixel, it is possible that a thin liquid film with a thickness of ${ }_{239}$ several micrometers was generated but was not observed on the upstream side of the droplet. 240

Owing to the very wide hysteresis region, the contact angles on the two sides of the ${ }_{241}$ droplet in our experiments differed significantly from each other. This difference induced 242 asymmetric capillary pressures on the two sides of the droplet and generated a pressure ${ }_{243}$ drop across the droplet that acted against the external pressure difference [12, 11]. The ${ }_{244}$ Poiseuille equation and the Young-Laplace equation should be valid for describing the rela- 245 tionships among the pressure drop across the droplet, the droplet velocity, and the contact 246 angles [2, 43, 11].

\subsubsection{Finger and film generation and film rupture}

The major difference between Flow Regime I and other flow regimes (Flow Regimes II, 249 III, IV) is the presence of liquid fingers and films. Fingers occur during the drainage process, 250 when a non-wetting liquid displaces a wetting liquid or when a liquid with low wettability ${ }_{251}$ displaces another liquid with high wettability under a partially wetting condition, even when 252 the viscosity of displacing liquid is much higher than the displaced liquid [44, 45, 12, 27, 13]. 253 Nevertheless, we observed no finger in Flow Regime I but a continuous liquid finger on the ${ }_{254}$ upstream side where drainage occurred in Flow Regime II, as shown in Figure 5(3). The 255 generation of liquid fingers during the drainage process should depend on the droplet velocity 256 or capillary number.

As the capillary number increased to $10^{-3}$ in Flow Regimes III and IV, the downstream 258 finger was also observable, as shown in Figure 6(4). Since the less viscous continuous liquid 259 


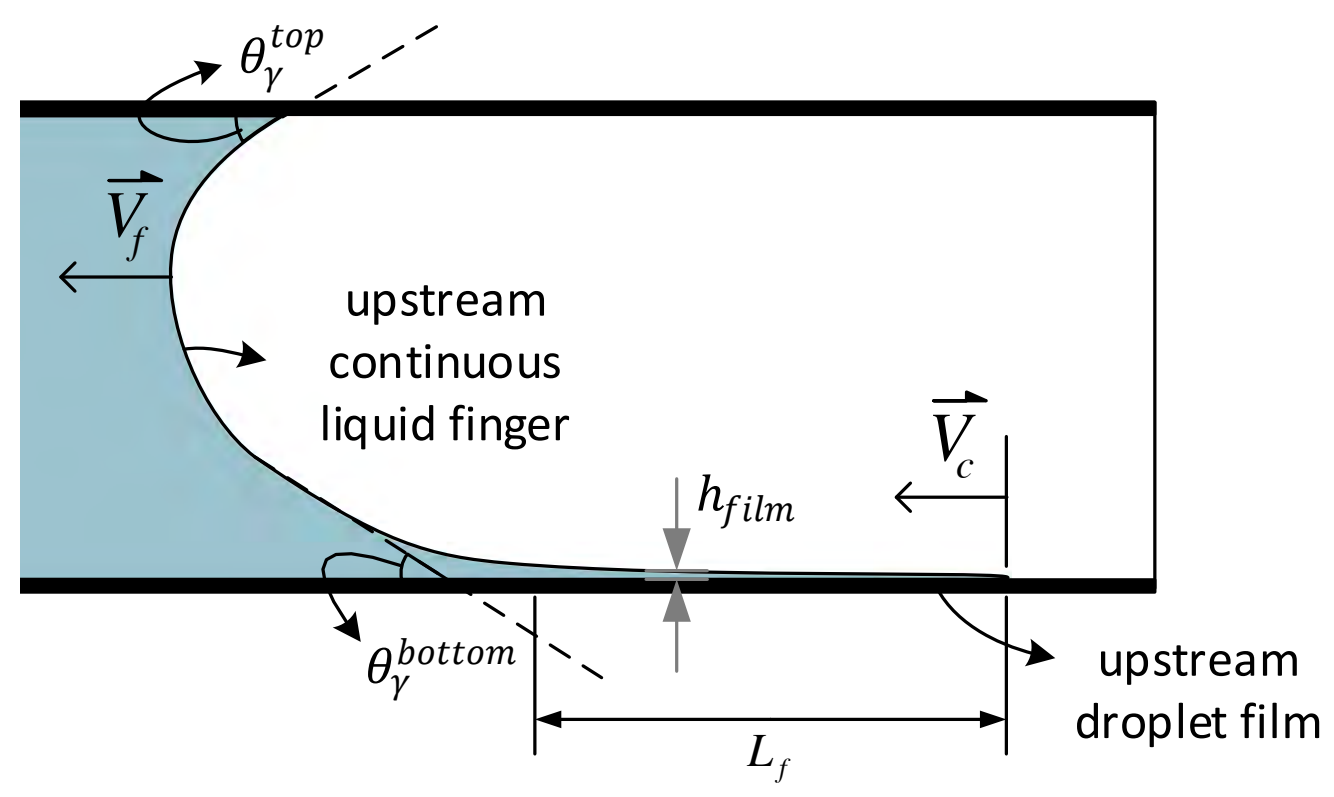

Figure 12: Schematic of the droplet moving in the Flow Regime II with the upstream continuous liquid finger and the droplet film deposited on the bottom of the tube wall, where $V_{c}$ is the velocity of the contact line on the upstream side, $V_{f}$ is the velocity of the interface, $L_{f i l m}$ is the length of the film, and $h_{f i l m}$ is the thickness of the film.

displaced more viscous droplet liquid on the upstream side, the downstream finger should 260 be associated with the Saffman-Taylor instability [37, 18, 13]. ${ }^{261}$

The thickness of films, $h_{\text {film }}$, is related to viscosity, interfacial tension, and droplet veloc- ${ }_{262}$ ity and is expected to be a function of capillary number [18, 1, 43]. In the current study, the ${ }_{263}$ thickness of the liquid films was asymmetric about the fingers because of the high density ${ }^{264}$ ratio of the droplet and the continuous liquids. In Flow Regime II, an apparent bottom 265 liquid film on the downstream side was detectable, but no top liquid film was detectable ${ }_{266}$ (see Figure 5). As capillary number increased in Flow Regimes III and IV, both top and ${ }_{267}$ bottom droplet liquid films were apparent on the upstream side, as shown in Figure $\mathbf{6}$ and 268 Figure 8. On the upstream side, the film on top was thinner than the film on bottom, and ${ }_{269}$ vice versa for the downstream side. In general, the asymmetry of the liquid films is critical 270 for droplet collapse and rolling, a topic that receives closer attention in sections 4.2 .3 and ${ }^{271}$ 4.2.4.

The contact lines in Flow Regime II and at the beginning of Flow Regimes III and IV ${ }_{273}$ were still sliding over the tube wall. However, the contact lines moved much slower than 274 
either the tip of the fingers or the major portion of the liquid-liquid interfaces. Therefore, 275 as shown in Figure 12, the length of the droplet and continuous liquid films, $L_{f i l m}$, grew 276 as the droplet moved. The growth rate depended on the difference between the finger tip 277 velocity $V_{f}$ and the contact line velocity $V_{c}$ :

$$
\frac{d L_{f i l m}}{d t}=V_{f}-V_{c}
$$

Actually, $V_{f}$ equaled the droplet velocity, and the growth rates of the liquid films were also ${ }_{279}$ the growth rates of the liquid fingers. In Flow Regime I, since the velocity of the contact line 280 equaled that of the liquid interface, no liquid film had been generated. In Flow Regime II, 281 the velocities of the contact lines of the bottom upstream droplet liquid films were $0.08 \mathrm{~mm} / \mathrm{s} 282$ $\left(Q_{t}=0.4 \mathrm{ml} / \mathrm{min}\right)$ and $0.09 \mathrm{~mm} / \mathrm{s}\left(Q_{t}=0.5 \mathrm{ml} / \mathrm{min}\right)$. By using Eq. (1), the growth rates of ${ }_{283}$ the upstream films were $0.12 \mathrm{~mm} / \mathrm{s}\left(Q_{t}=0.4 \mathrm{ml} / \mathrm{min}\right)$ and $0.28 \mathrm{~mm} / \mathrm{s}\left(Q_{t}=0.5 \mathrm{ml} / \mathrm{min}\right)$. ${ }^{284}$ In Flow Regimes III and IV during the short period before droplet collapse and rolling, the 285 velocities of the contact lines were $0.12 \mathrm{~mm} / \mathrm{s}\left(Q_{t}=0.6 \mathrm{ml} / \mathrm{min}\right)$ and $0.10 \mathrm{~mm} / \mathrm{s}\left(Q_{t}=0.8{ }^{286}\right.$ $\mathrm{ml} / \mathrm{min})$, and the growth rates of the upstream films were $0.41 \mathrm{~mm} / \mathrm{s}\left(Q_{t}=0.6 \mathrm{ml} / \mathrm{min}\right){ }^{287}$ and $0.61 \mathrm{~mm} / \mathrm{s}\left(Q_{t}=0.8 \mathrm{ml} / \mathrm{min}\right)$. As capillary number increased, the growth rate of liquid ${ }_{288}$ films clearly increased, but the increase of $V_{c}$ was non-significant. It appears that when the ${ }_{289}$ liquid films were generated, the increase of the contact line velocity-owing to the increase in 290 capillary number-became very limited. 291

The thin liquid films were unstable and ruptured as the droplet moved forward, as shown 292 in Figure 5(3). The film rupture was likely linked to the instability of the liquid interface ${ }_{293}$ of the thin liquid film along the solid boundary. In general, the occurrence of film rupturing 294 depends on the thickness of film, density ratio, droplet velocity, and the interfacial tension 295 between the two given liquids [46]. 296

In Flow Regime II, an apparent droplet liquid film was formed on the upstream side, as 297 shown in Figure 5(1). Then, the film ruptured, a new sliding contact line of the moving 298 droplet was immediately formed, and a certain amount of the droplet liquid was left behind 299 the moving droplet. The locations of the film rupture for $Q_{t}=0.4 \mathrm{ml} / \mathrm{min}$ and $Q_{t}=0.5 \quad 300$ 
$\mathrm{ml} / \mathrm{min}$ were close, but the amount of droplet liquid left behind the moving droplet for 301 $Q_{t}=0.5 \mathrm{ml} / \mathrm{min}$ was slightly larger than for $Q_{t}=0.4 \mathrm{ml} / \mathrm{min}$. We also found that very 302 thin films were generated above the upstream fingers, and the films ruptured much earlier 303 than the bottom ones for both $Q_{t}=0.4 \mathrm{ml} / \mathrm{min}$ and $Q_{t}=0.5 \mathrm{ml} / \mathrm{min}$. No film rupture was ${ }^{304}$ observed in Flow Regime III, but the amount of the leftover droplet liquid in Flow Regime IV 305 was much larger than the ones in Flow Regime II. The amount of droplet liquid left behind 306 such a moving droplet should, in general, be related to the thickness of the upstream film as 307 well as the capillary number.

\subsubsection{Finger-induced droplet collapse}

308

The major difference in droplet behavior between Flow Regime II and Flow Regimes III 310 and IV is related to the presence of the "downstream" finger and film, as shown in Fig- 311 ure 6(4). The upstream finger eventually met with the downstream film at points A and 312 B, as shown in Figure 8(4) (solid line). Before the collapse, the droplet was still connected 313 with the downstream droplet liquid film by thin droplet liquid layers at point A and B. 314 However, the thin liquid layer was unstable and easily breakable owing to Plateau-Rayleigh 315 instability [47].

Because the droplet liquid was denser than the continuous liquid, the dropletliquid film 317 along the continuous liquid finger was asymmetric owing to the effect of gravity. As shown 318 in Figure 8(4) (solid line), the top upstream liquid layer at point A was much thinner than ${ }_{319}$ the bottom one at point B. As the droplet moved forward, the droplet liquid layer broke at 320 point A, and the surrounding liquid freely flowed above the droplet. Meanwhile, the liquid ${ }^{321}$ droplet layer at point B was too thick to be broken, the whole droplet was not able to escape 322 from the tube wall and then collapsed. A mobilized droplet turned into an immobile one. ${ }^{323}$ This may happen even if the external pressure gradient is larger than the critical pressure ${ }^{324}$ gradient for droplet mobilization that is predicted by theory based on the Young-Laplace 325 equation [19. 
In Flow Regime IV, the dropletbehaved similarly to the droplet in Flow Regime III, 328 initially moving while forming fingers on both the downstream and the upstream sides. No 329 film rupture occurred before the rolling. At the critical moment just before rolling, as shown 330 in Figure 8(4) (dashed line), the layer at point B was thinner than the corresponding layer 331 in Flow Regime III. As a result, the droplet liquid layer at point B broke apart immediately 332 after the breakup of the droplet liquid layer at point A. Instead of droplet collapse, the 333 droplet snapped off from the droplet liquid films on the capillary tube and rolled forward 334 with an increased velocity.

We added silver particles to the droplet liquid to reveal the flow pattern inside the 336 droplet before and after rolling. Figure 13 schematically shows the observed pre-rolling and 337 post-rolling flow patterns, constructed by means of the relative particle velocities. The term 338 'relative particle velocity' is here defined as the velocity of a particle relative to the velocity ${ }_{339}$ of the moving liquid-liquid interfaces. In the current study, all liquids and particles moved 340 from right to left. The arrows in Figure $\mathbf{1 3}$ represent the direction of particle movement ${ }_{341}$ relative to the interfaces. Arrows pointing to the left show the particles that move faster 342 than the interfaces, and arrows pointing to the right show the particles that move slower ${ }_{343}$ than the interfaces. Before rolling, the flow velocity in the middle of the droplet was larger 344 than the interface velocity, while the flow velocity near the tube wall was slower than the 345 interface velocity. After the rolling, the particles in the upper part of the droplet moved 346 faster than the droplet, while the ones in the lower part of the droplet moved slower than ${ }_{347}$ the droplet. A vortex was clearly formed inside the droplet, and the whole droplet rolled 348 forward.

The mechanism for shifting droplet movement from a slug motion to a rolling motion is 350 related to asymmetric thin-layer breakup. As shown in Figure 14(1), when the thin layer 351 at point A broke, it induced a jet parallel to the flow direction to the top of the droplet to 352 trigger the rolling motion. Then, the breakup of the thin layer at point B induced an upward 353 jet to enhance the rolling motion. 


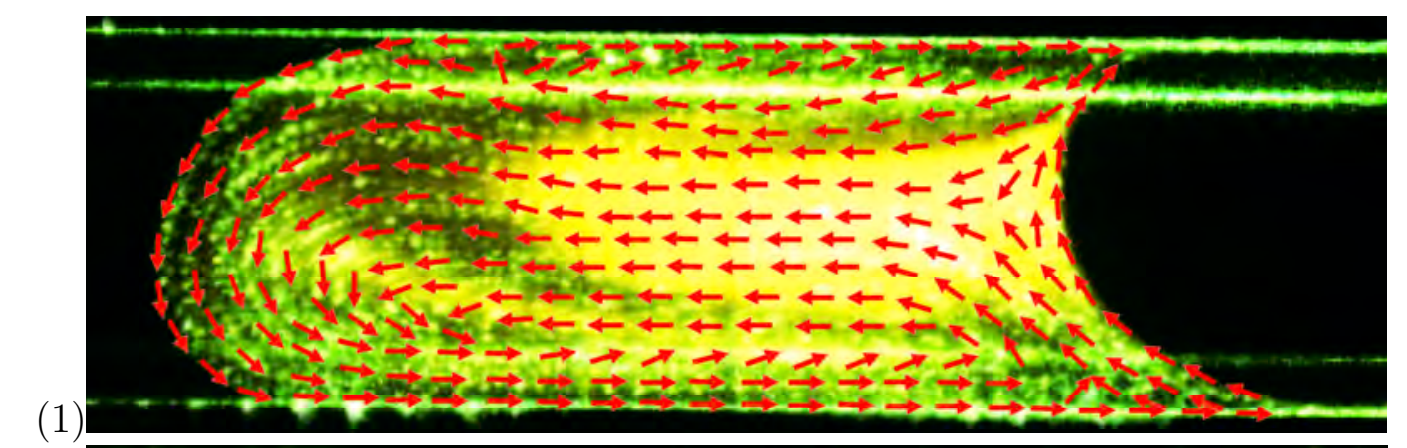

(1)

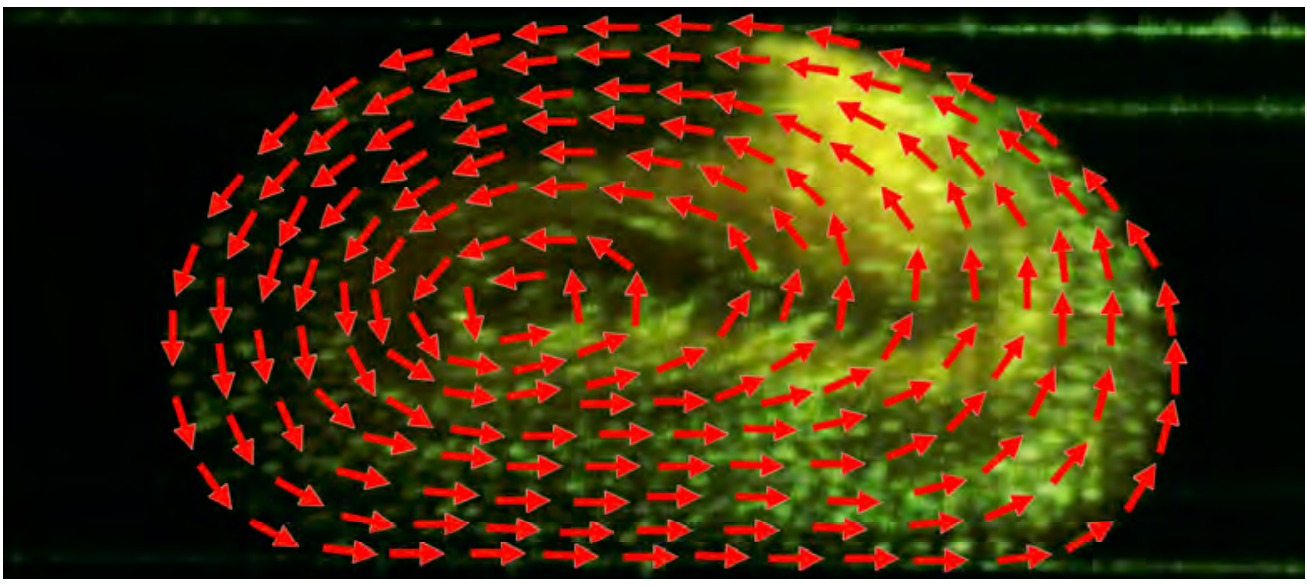

Figure 13: Schematic flow patterns inferred from observing particle motion relative to the liquid-liquid interfaces shown for (1) before the droplet rolling and (2) after the droplet rolling. The red arrows represent the direction of particle movement relative to the liquid-liquid interfaces, but the size of arrows shown does not reflect the magnitudes of the particle velocities. 


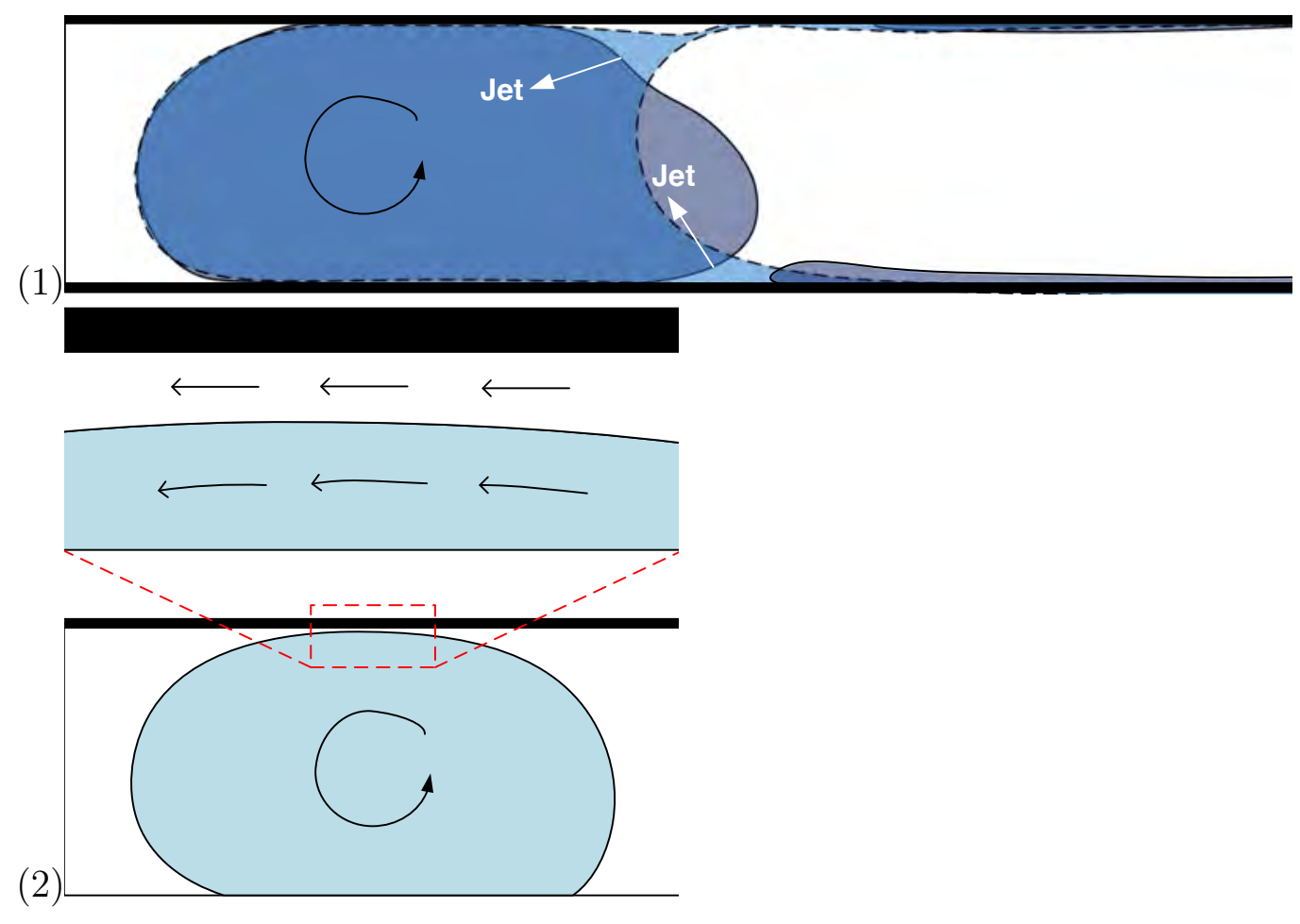

Figure 14: Schematic of the droplet rolling mechanism in the Flow Regime IV. (1) The rolling motion is triggered by two jets when the liquid layers were broken. (2) The rolling motion is triggered due to the fast continuous liquid flow above the droplet.

Another possible mechanism is due to the flow of the continuous liquid above the droplet af- 355 ter rolling. As shown in Figure 14(2), when the droplet liquid layer at point A broke, the 356 less-dense continuous liquid passed fast through the small channel above the droplet and ${ }_{357}$ drove the top droplet liquid through viscous force. The flow velocity difference between the 358 top and bottom of the droplet generated a vortex inside the droplet and made the droplet roll 359 forward.

After the rolling, the change in interfacial curvature largely decreased the capillary pres- 361 sure across the upstream interface. The decrease in capillary pressure reduced the pressure 362 drop sustained by the droplet and therefore enhanced droplet movement. It appears as 363 though Flow Regime IV and the decrease of the pressure drop were also observed by Dong 364 et al. [12], who examined oil droplet mobilization in a capillary tube, even though they 365 did not comment on the droplet rolling, which we observed. Even so, they showed that, 366 during droplet mobilization, the pressure drop that the droplet sustained increased at the 367 
beginning of the process, when the liquid-liquid interfacial curvature gradually changed, and 368 then dropped drastically when the sign of the interfacial curvature of the oil droplet changed, 369 and the oil droplet was completely surrounded by water. In addition, after the rolling, the 370 droplet rolled without dragging the droplet liquid films along the capillary tube wall. Ap- 371 parently, the change in the droplet motion from sliding to rolling is another reason for the 372 increased droplet moving velocity.

A rolling non-wetting liquid droplet is related to the cleaning mechanism of a water 374 droplet on biological and artificial surfaces. In a classical figure of Barthlott's paper, only 375 a rolling water droplet -not a sliding one-can serve to remove particles (dirt) [48]. A water ${ }_{376}$ droplet can roll on a lotus leaf owing to the large roughness of the leaf. This is referred to as 377 the lotus effect, or roughness-induced non-wetting [48, 16]. However, the rolling droplet in 378 our experiments was initially in the partially wetting condition, and, without changes to 379 surface roughness or surface wettability, finger-induced rolling can dynamically transition 380 the droplet into non-wetting regime.

\subsection{Pressure drops in the flow regimes}

The Young-Laplace equation and the Hagen-Poiseuille equation are commonly used for 383 calculating the capillary and viscous pressure drops in predicting the pressure drops across 384 a trapped droplet as well as in dynamic network modeling of multiphase flow in porous 385 media [2, 49, 19, 50, 51, 52, 53, 54]. The pressure drop across the pore doublet based on the 386 Young-Laplace equation and the Hagen-Poiseuille equation is

$$
\Delta P_{\text {pre }}=\underbrace{2 \sigma\left[\frac{\cos \left(\theta_{A}^{(d y n)}\right)}{r_{1}-h_{A}^{f i l m}}-\frac{\cos \left(\theta_{R}^{(d y n)}\right)}{r_{1}-h_{R}^{f i l m}}\right]}_{\text {capillary pressure }}+\underbrace{\frac{8 \eta_{d} V_{d}}{r_{1}^{2}} L_{1 d}+\frac{8 \eta_{c} V_{d}}{r_{1}^{2}} L_{1 c}}_{\text {viscous pressure drop }}
$$

where $V_{d}$ is the droplet velocity, $r_{1}$ is the radius of Tube $1, L_{1 c}$ is the total length of the ${ }_{388}$ continuous liquid in Tube $1, L_{1 d}$ is the length of the droplet in Tube $1, \eta_{c}$ is the viscosity 389 of the continuous liquid, $\eta_{d}$ is the viscosity of the droplet liquid, $\theta_{A}^{(d y n)}$ and $\theta_{R}^{(d y n)}$ are the 390 dynamic contact angles of the advancing and receding interfaces, and $h_{A}^{f i l m}$ and $h_{R}^{f i l m}$ are ${ }_{391}$ the thickness of the liquid films on the advancing and receding interfaces [43]. A recent ${ }_{392}$ 
study has shown that Eq. (2) can help adequately model the fate of trapped droplets and 393 droplets moving with a very low velocity and with no liquid films (i.e., $h_{A / R}^{f i l m}=0$ ) [11]. ${ }^{394}$

We directly measured the pressure drops, $\Delta P_{m}$, across the pore doublet with pressure 395 transducers P1 and P2, as shown in Figure 2. Figure 15shows that $\Delta P_{m}$ increased as $Q_{t}{ }_{396}$ increased. Moreover, $\Delta P_{\text {pre }}$ from Eq. (2) is about the same as $\Delta P_{m}$ for $Q_{t}=0.2 \mathrm{ml} / \mathrm{min}$ in ${ }_{397}$ Flow Regime I. However, the deviation between $\Delta P_{p r e}$ and $\Delta P_{m}$ drastically increased from 398 Flow Regime I to Flow Regime II and III in which liquid films were generated, and it jumped 399 up again in Flow Regime IV.

We measured the large pressure deviations, $\Delta P_{m}-\Delta P_{\text {pre }}$, when mobilizing an oil slug in 401 a straight capillary filled with water [12]. The causes of the extra pressure drops are likely 402 linked to (1) the internal flow within the moving droplet, (2) the movement of the sliding ${ }_{403}$ contact line, and (3) the generation of the liquid films [12]. The fall of the extra pressure ${ }_{404}$ drop after the rolling was related to the deformation of the liquid interface on the upstream 405 side and the change of the flow pattern inside the droplet (i.e., from a slug flow to a rolling 406 flow). The extra pressure drops are significant comparing to the capillary pressure and the ${ }_{407}$ viscous pressure drops during droplet mobilization. However, up to the present moment, no ${ }_{408}$ sound theory is available for satisfactorily predicting the extra pressure drop. 409

\subsection{Effects of gravity and pore geometry on the occurrence of flow regimes 410}

According to studies, the asymmetry that gravitational force can cause in the liquid ${ }_{411}$ films around a non-wetting droplet in horizontal capillary tubes is significant for large Bond ${ }_{412}$ numbers, $B o=\left(\Delta \rho g R^{2}\right) / \gamma$, where $R$ is the radius of the capillary tube [55, 56]. The Bond ${ }_{413}$ number is defined as the ratio of a typical gravity to a typical capillary force. Gu and Yang ${ }^{414}$ [33] derived a theoretical model to show that a Bond number has a strong effect on the ${ }_{415}$ shape of interfacial profiles when the value of the Bond number is larger than 0.025. The ${ }^{416}$ Bond number of our experimental setup was approximately 1, and the effect of gravity on ${ }_{417}$ the interfacial profile and film thickness was pronounced and resulted in droplet collapse in ${ }_{418}$ the Flow Regime III.

Sharon et al. [56] showed that the level of the liquid film asymmetry around a moving non- ${ }_{420}$ 


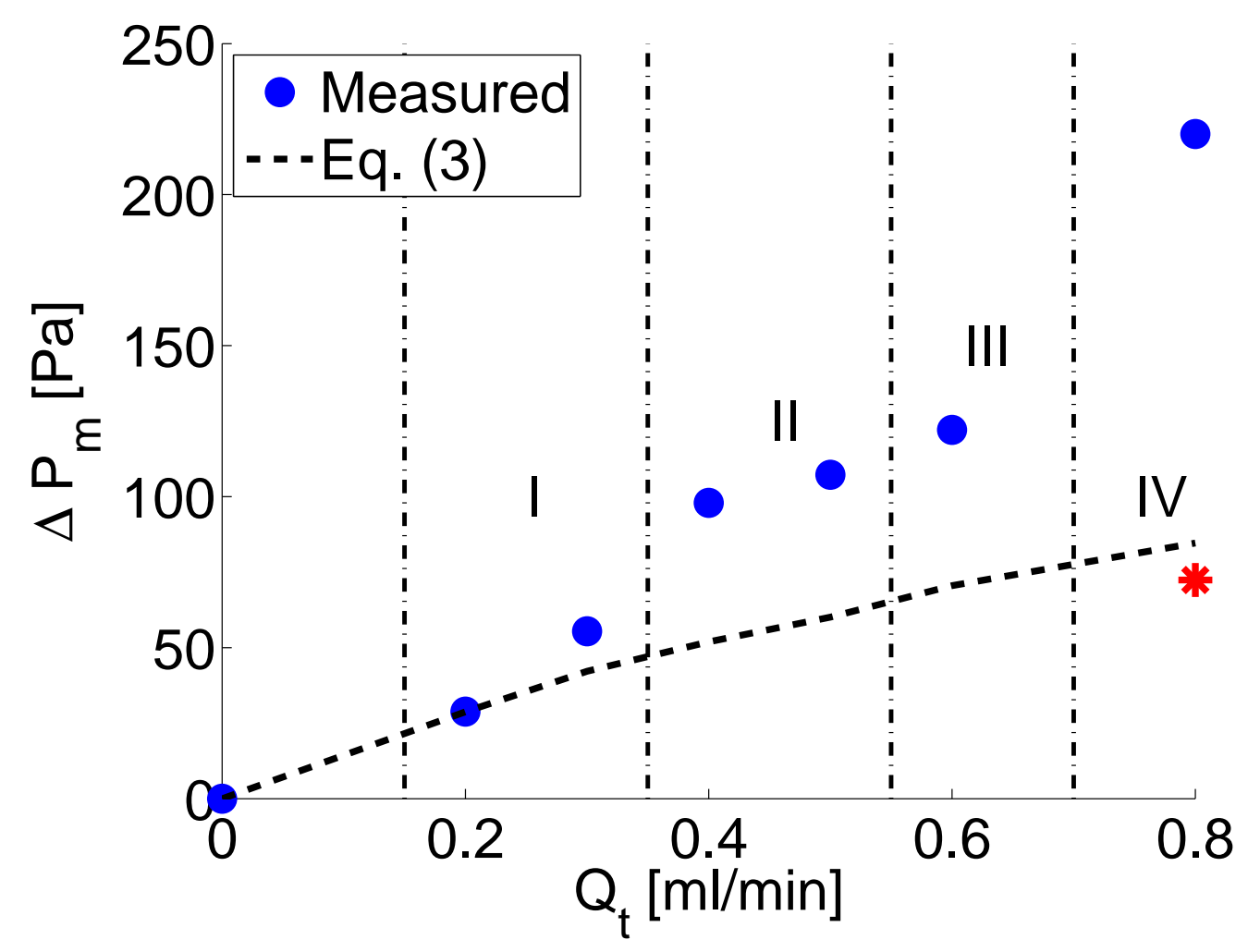

Figure 15: The measured and predicted pressure drops across the pore doublet model versus pumping rate $Q_{t}$. The star represents pressure drop after droplet rolling. The predicted pressure drop is calculated from Eq. (2) with measured contact angles. 
wetting droplet can decline as the capillary number increases. Their finding could explain ${ }^{421}$ the different droplet behaviors in Flow Regimes III and IV. Thus, in our current study, as ${ }_{422}$ capillary number increased, the liquid films in Flow Regime IV were less asymmetric than ${ }_{423}$ those in Flow Regime III. Therefore, at the critical moment just before rolling, as shown ${ }_{424}$ in Figure 8(4)(dashed line), the film thickness at point B was about the same as the film ${ }_{425}$ thickness at point A. As a result, the droplet liquid layer at point A and B broke apart ${ }_{426}$ almost at the same time, and, instead of droplet collapse, the droplet rolled forward with ${ }_{427}$ an increased velocity. We can reasonably conclude that, in general, Flow Regime III might ${ }_{428}$ occur only when the effect of gravity is important (large Bond number); otherwise, at a ${ }^{429}$ high capillary number regime, Flow Regime IV is likely to be common. Furthermore, the ${ }^{430}$ asymmetric film thickness is a possible mechanism in the triggering of droplet rolling. A ${ }_{431}$ definitive boundary between Flow Regimes III and IV or a phase diagram of them should ${ }_{432}$ depend on both Bond number and capillary number.

The flow regimes observed in our experiments could also occur in the pore geometries ${ }_{434}$ different from a straight tube of circular cross-section which has been applied in our experi- ${ }_{435}$ ment. Oskooei and Sinton [27] showed that a partial wetting droplet can move with a sliding ${ }^{436}$ contact line in a rectangular channel. Recently, Jose and Cubaud [28] demonstrated that a ${ }^{437}$ partially wetting droplet can move with contact lines along a square channel surface as long ${ }_{438}$ as the diameter of the droplet is larger than the height of the channel. In their study, a thin ${ }^{439}$ film between the tube wall and the droplet formed when the capillary number increased. ${ }_{440}$ Although their visualization method's limitations prevented any observation of films and ${ }_{441}$ fingers around the moving droplet, the aforementioned study has shown that a droplet can ${ }_{442}$ move with and without moving contact lines in capillary tubes of angular cross-section. It ${ }_{443}$ is reasonable to expect that Flow Regimes III and IV will occur at a high Bond number. In ${ }_{444}$ addition, in capillary tubes of angular cross-section, the thick films formed in corners should ${ }_{445}$ have less viscous pressure drops than was the case with the very thin films formed in our ${ }_{446}$ experiments. The extra pressure drops measured across the droplet in our study perhaps ${ }_{447}$ could have been smaller in capillary tubes of angular cross-section than in those of circular ${ }_{448}$ 
cross-section.

It took a certain travel distance for a partial wetting droplet to develop the mecha- ${ }_{450}$ nisms of the droplet rolling and droplet collapse. Rolling and collapse occurred when an ${ }_{451}$ upstream meniscus reached the downstream contact points. If the contact points are com- ${ }^{452}$ pletely pinned, the "development length" could theoretically be as short as the droplet length. ${ }_{453}$ It might also depend on how long the thin layer, which connects the droplet film to the ${ }_{454}$ droplet, can persist before breaking up. The "development lengths" of the droplet rolling and 455 droplet collapse observed in our experiments were both around $7 \mathrm{~mm}$, which is about twice ${ }_{456}$ the length of the droplet, as shown in Figures 6 and 8. Droplet rolling and droplet collapse ${ }_{457}$ might not occur if the length of a straight capillary tube is less than the given "development ${ }_{458}$ lengths". Nevertheless, the pore channels are not necessarily be straight, as established by ${ }_{459}$ Payatakes [57]: a finger or ganglion fully surrounded by the continuous liquid can freely move 460 in tortuous channels. The length of a tortuous channel in a real geological porous media can ${ }_{461}$ easily be much longer than the "development lengths" of droplet rolling and droplet collapse. ${ }_{462}$ However, a channel of such length would not have the same cross-section along its length ${ }_{463}$ like the capillary tube used in our experiment. The variation in the radius of the channel ${ }_{464}$ would likely affect droplet rolling and collapse. Further investigation into the effects of pore ${ }_{465}$ geometry on the occurrence of the observed flow regimes is necessary to clarify the potential ${ }_{466}$ for the practical applications of our findings.

\subsection{Impact of different flow regimes on network modeling, groundwater reme- ${ }_{468}$ diation, oil recovery and geological carbon sequestration (GCS)

The current study's results show that, because of the presence of liquid fingers and films, 470 the pressure drop across the moving droplet in Flow Regimes II, III, and IV is significantly ${ }^{471}$ higher than the predictions based on Poiseuille and Young-Laplace equations. Although ${ }^{472}$ the extra pressure drop was obtained in a straight tube, it is obvious that the presence of ${ }^{473}$ liquid fingers and films could decrease the mobility of residual droplets in porous media ${ }_{474}$ during the practical applications, such as enhanced oil recovery or groundwater remediation. 475 Furthermore, due to the rupture of the film formed by the droplet liquid, a certain amount 476 of the droplet liquid can be left behind a moving droplet. In the context of groundwater ${ }_{477}$ 
remediation, the remaining NAPL droplet is difficult to be remobilized. However, the small 478 NAPL droplet should readily dissolve because of its large specific surface area. Therefore, a 479 higher pumping rate by establishing Flow Regime II or IV instead of I, might not lead to a 480 $\begin{array}{ll} & 481\end{array}$

On the other hand, capillary trapping is also an important mechanism upon which GCS ${ }_{482}$ relies. Recent studies have found that, during the process of GCS, the $\mathrm{CO}_{2}$ can partially wet ${ }_{483}^{4}$ mineral surfaces [58, 42]. The stability of trapped partially wetting $\mathrm{CO}_{2}$ droplets is related ${ }_{484}$ to the pressure gradients that the $\mathrm{CO}_{2}$ droplets can sustain. The occurrence of liquid films ${ }_{485}$ or fingers increases the pressure drops across the droplet and slows down moving droplets. ${ }_{486}$ This phenomenon should result in enhanced GCS storage if a reservoir experiences flow with ${ }_{487}$ capillary numbers from Flow Regimes II and III. 488

We have shown that the occurrence of Flow Regimes III and IV highly depends on ${ }_{489}$ the Bond number. Flow at a large Bond number could occur when the enhanced oil re- 490 covery or groundwater remediation based on surfactant flushing serves to remobilize dense ${ }_{491}$ droplets non-aqueous phase or light non-aqueous phase droplets, such as PCE or oils. Owing 492 to both the low interfacial tensions as low as $10^{-1}$ dyne $/ \mathrm{cm}$ and the density differences up to ${ }_{493}$ $1 \mathrm{~g} / \mathrm{cm}^{3}$, the Bond numbers could exceed $10^{-2}$. The Bond number could increase drastically 494 when the droplets are trapped in large pores and fractures. Similarly, as the supercritical 495 $\mathrm{CO}_{2}$ is trapped in large pores or fractures, the small density ratio of supercritical $\mathrm{CO}_{2}$ to ${ }_{496}$ surrounding brine might result under flow conditions characterized by high Bond numbers. ${ }^{497}$ The occurrence of Flow Regimes III and IV is also related to the stability of supercritical 498 $\mathrm{CO}_{2}$ entrapment. The four flow regimes observed in this manuscript could be related to ${ }_{499}$ $\begin{array}{ll}\text { practical applications. } & 500\end{array}$

\section{Conclusions 201}

In this study, we have visualized the mobilization of a partially wetting droplet in a 502 capillary tube of a pore doublet under different flow conditions. Four flow regimes have 503 been observed for steady background flows. Droplets moved with or without forming liquid 504 fingers or films. We found that mobilization can be terminated by droplet collapse or can 505 
be dramatically enhanced if a given droplet snaps off from the tube wall and rolls forward. 506

Our results show that the generation of the liquid films and fingers is highly related to 507 capillary number. The higher the capillary number, the thicker the liquid films. During the 508 droplet movement in Flow Regime II, a certain amount of droplet liquid was left behind 509 the main droplet owing to an upstream film rupture. The gravity-induced asymmetry of 510 downstream liquid films caused the collapse of the moving droplet. However, by increas- 511 ing the droplet velocity, the degree of the asymmetry of the films was reduced, and the 512 droplet snapped off from the tube wall. The change of the interface shape and the flow pat- ${ }_{513}$ tern inside the droplet after rolling reduced the pressure drop sustained by the droplet and 514 increased the droplet velocity. 515

Finally, the formation of droplet and continuous liquid films might hinder oil extraction 516 and groundwater remediation based on pump-and-treat and flooding methods. In contrast, 517 liquid films should enhance the stability of capillary trapped $\mathrm{CO}_{2}$ droplets. 518

In this manuscript, we focused on introducing of four flow regimes of a partially wetting 519 droplet in capillaries. Owing to the limitations of the visualization technique, our findings 520 is based on limited experiments for high Bond number. The effects of the liquid properties 521 and pore geometry on the occurrence of these flow regimes have not yet been carefully 522 explored. This study's discovery should lead to additional work investigating the complicated 523 mechanics of droplet mobilization in a partial wetting regime within different flow conditions. $\quad 524$

\section{Acknowledgments ${ }_{525}$}

This work was supported by NSF Grant EAR-0739038, (USA) and NSC Grant 102-2116- 526 M-008-002, (ROC), Taiwan. 527

\section{References ${ }^{528}$}

[1] G. I. Taylor, Deposition of a viscous fluid on the wall of a tube, Journal of Fluid ${ }_{529}$ Mechanics 10 (1961) 161-165. 530

[2] W. Rose, R. W. Heins, Moving interfaces and contact angle rate-dependency, Journal ${ }_{531}$ of Colloid Science 17 (1) (1962) 39-48. 532 
[3] R. L. Hoffman, A study of the advancing interface, Journal of Colloid and Interface ${ }_{533}$ Science 50 (2) (1975) 228-241.

[4] K. M. Ng, H. T. Davis, L. E. Scriven, Visualization of blob mechanics in flow through 535 porous media, Chemical Engineering Science 33 (8) (1978) 1009-1017.

[5] J. L. Wilson, Pore scale behavior of spreading and non-spreading organic liquids in the ${ }_{537}$ vadose zone, in: K. U. Weyer (Ed.), Subsurface Contamination by Immiscible Fluids, ${ }_{538}$ A.A. Balkema, Rotterdam, 1992.

[6] S. H. Conrad, J. L. Wilson, W. R. Mason, W. J. Peplinski, Visualization of residual 540 organic liquid trapped in aquifers, Water Resources Research 28 (2) (1992) 467-478. $\quad{ }^{541}$

[7] E. Schäffer, P. Z. Wong, Dynamics of contact line pinning in capillary rise and fall, 542 Physical Review Letters 80 (14) (1998) 3069-3072.

[8] M. Hilpert, Effects of dynamic contact angle on liquid infiltration into horizontal capil- ${ }^{544}$ lary tubes: (semi)-analytical solutions, Journal of Colloid and Interface Science 337 (1) 545 (2009) 131-137.

[9] I. A. Beresnev, A. Gaul, R. D. Vigil, Direct pore-level observation of permeability 547 increase in two-phase flow by shaking, Geophysical Research Letters 38 (2011) L20302. ${ }_{548}$ doi:10.1029/2011GL048840.

[10] N. R. Morrow, Wettability and its effect on oil recovery, Journal of Petroleum Technol- 550 ogy 42 (12) (1990) 1476-1484.

[11] S. Y. Hsu, R. Glantz, M. Hilpert, Pore-scale analysis of the effects of contact angle ${ }_{552}$ hysteresis on blob mobilization in a pore doublet, International Journal of Oil, Gas and ${ }_{553}$ Coal Technology 5 (2012) 207-228.

554

[12] M. Dong, Q. Fan, L. Dai, An experimental study of mobilization and creeping flow 555 of oil slugs in a water-filled capillary, Transport in Porous Media 80 (2009) 455-467. 556 doi:10.1007/s1122-009-9374-5. 
[13] L. Cueto-Felgueroso, R. Juanes, Macroscopic phase-field model of partial wetting: bub- ${ }_{558}$ bles in a capillary tube, Physical Review Letters 108 (2012) 144502. doi:10.1103/ 559 PhysRevLett.108.144502.

[14] L. Leger, J. F. Joanny, Liquid spreading, Reports on Progress in Physics 55 (1992) 431. 561 doi:10.1088/0034-4885/55/4/001.

[15] R. R. Netz, D. Andelman, Roughness-induced wetting, Physical Review E 55 (1) (1997) ${ }_{563}$ 687.

[16] S. Herminghaus, Roughness-induced non-wetting, Europhysics letters 52 (2) (2000) 165- 565 170.

[17] J. Bico, C. Tordeux, D. Quéré, Rough wetting, EPL (Europhysics Letters) 55 (2) (2001) 567 214.

[18] F. P. Bretherton, The motion of long bubbles in tubes, Journal of Fluid Mechanics 10569 (1961) 166-188.

[19] J. C. Melrose, C. F. Brandner, Role of capillary force in determining microscopic dis- ${ }^{571}$ placement efficiency for oil recovery by waterflooding, Can. J. Petrol. Technol. 13 (4) ${ }^{572}$ (1974) 54-62.

[20] P. G. de Gennes, Wetting: Statics and dynamics, Reviews of Modern Physics 57 (3, 574 part 1) (1985) 827-863.

[21] P. Thompson, M. Robbins, Simulations of contact-line motion: slip and the dynamic 576 contact angle, Physical Review Letters 63 (7) (1989) 766-769.

[22] P. Joos, P. van Remoortere, M. Bracke, The kinetics of wetting in a capillary, Journal 578 of Colloid and Interface Science 136 (1) (1989) 189-197.

[23] J. Joanny, M. Robbins, Motion of a contact line on a heterogeneous surface, The Journal 580 of Chemical Physics 92 (1990) 3206-3212. 
[24] M. Martinez, K. S. Udell, Axisymmetric creeping motion of drops through circular 582 tubes, Journal of Fluid Mechanics 210 (2012) 565-591. 583

[25] P. Aussillous, D. Quéré, Quick deposition of a fluid on the wall of a tube, 584 Physics of Fluids (1994-present) 12 (10) (2000) 2367-2371. doi:http: 585 //dx.doi.org/10.1063/1.1289396. 586 URL http://scitation.aip.org/content/aip/journal/pof2/12/10/10.1063/1. 587 1289396

[26] D. Bonn, J. Eggers, J. Indekeu, J. Meunier, E. Rolley, Wetting and spreading, Reviews 589 of modern physics 81 (2009) 739-805. doi:10.1103/RevModPhys.81.739. 590

[27] S. A. K. Oskooei, D. Sinton, Partial wetting gas-liquid segmented flow microreactor, 591 Lab on a Chip 10 (2010) 1732-1734. 592

[28] B. M. Jose, T. Cubaud, Formation and dynamics of partially wetting droplets in square 593 microchannels, RSC Adv. 4 (2014) 14962-14970. doi:10.1039/C4RA00654B. 594 URL http://dx.doi.org/10.1039/C4RA00654B

[29] X. Luo, X.-P. Wang, T. Qian, P. Sheng, Moving contact line over undulating surfaces, 596 Solid state communications 139 (2006) 623-629.

[30] I. Chatzis, F. A. L. Dullien, Dynamic immiscible displacement mechanisms in pore 598 doublets: theory versus experiment, Journal de Physique II 2 (11) (1992) 2025-2038. 599

[31] C. D. Montemagno, W. G. Gray, Photoluminescent volumetric imaging: a technique for ${ }_{600}$ the exploration of multiphase flow and transport in porous media, Geophysical Research 601 Letters 22 (4) (1995) 425-428.

[32] M. R. Sasges, C. A. Ward, Effect of gravity on contact angle: an experimental investiga- ${ }_{603}$ tion, Journal of Chemical Physics 109 (9) (1998) 3661-3670. doi:10.1063/1.476962. 604 
[33] Y. Gu, C. Yang, The effects of capillary force and gravity on the interfacial profile in a ${ }_{605}$ reservoir fracture or pore, Journal of Petroleum Science \& Engineering 40 (1-2) (2003) 606 77-87.

[34] E. B. Dussan V., On the spreading of liquids on solid surfaces: static and dynamic ${ }_{608}$ contact lines, Annual Review Fluid Mechanics 11 (1979) 371-400.

[35] V. S. Averbakh, S. N. Vlasov, Y. M. Zaslavsky, Motion of a liquid droplet in a capil- ${ }_{610}$ lary under the action of static force and an acoustic field, Radiophysics and Quantum 611 Electronics 43 (2) (2000) 142-147.

[36] H. B. Eral, D. J. C. M. t. Mannetje, J. M. Oh, Contact angle hysteresis: a review of ${ }_{613}$ fundamentals and applications, Colloid and polymer science 291 (2) (2013) 247-260. ${ }_{614}$

[37] P. G. Saffman, G. Taylor, The penetration of a fluid into a porous medium or hele-shaw ${ }_{615}$ cell containing a more viscous liquid, Proc. R. Soc. London, Ser. A 245 (1242) (1958) 616 $312-329$.

[38] P. van Remoortere, P. Joos, The kinetics of wetting: the motion of a three phase ${ }_{618}$ contactline in a capillary, Journal of Colloid and Interface Science 141 (2).

[39] L. Tanguy, T. Leïchlé, L. Nicu, Dynamic spreading of a liquid finger driven by elec- ${ }_{620}$ trowetting: Theory and experimental validation, Journal of applied physics 101 (2007) ${ }_{621}$ 044907.

[40] E. Schäffer, P. z. Wong, Contact line dynamics near the pinning threshold: A capillary ${ }_{623}$ rise and fall experiment, Physical Review E 61 (5) (2000) 5257-5277.

[41] J. Bico, U. Thiele, D. Quéré, Wetting of textured surfaces, Colloids and Surfaces A: ${ }_{625}$ Physicochemical and Engineering Aspects 206 (2002) 41-46.

626

[42] Y. S. Jun, D. E. Giammar, C. J. Werth, Impacts of geochemical reactions on geologic ${ }_{627}$ carbon sequestration, Environmental Science \& Technology 47 (1) (2013) 3-8. doi: 628 10.1021/es3027133. 
[43] J. Bico, D. Quéré, Falling slugs, Journal of Colloid and Interface Science 243 (2001) ${ }_{630}$ 262-264.

[44] M. T. Kreutzer, F. Kapteijn, J. A. Moulijn, J. J. Heiszwolf, Multiphase monolith re- 632 actors: chemical reaction engineering of segmented flow in microchannels, Chemical ${ }_{633}$ Engineering Science 60 (2005) 5895-5916.

[45] C. N. Baroud, M. R. S. Vincent, D. J-P, An optical toolbox for total control of droplet ${ }_{635}$ microfluidics, Lab on a Chip 7 (2007) 1029-1033.

[46] A. Oron, S. Davis, S. G. Bankoff, Long-scale evolution of thin liquid films, Reviews of ${ }_{637}$ Modern Physics 69 (3) (1997) 931-980.

[47] J. Eggers, Nonlinear dynamics and breakup of free-surface flows, Reviews of Modern ${ }_{639}$ Physics 69 (3) (1997) 865-929.

[48] W. Barthlott, C. Neinhuis, Purity of the scared lotus, or escape from contamination in ${ }_{641}$ biological surfaces, Planta 202 (1997) 1-8.

[49] J. J. Taber, Dynamic and static forces required to remove a discontinuous oil phase ${ }_{643}$ from porous media containing both oil and water, Soc. Pet. Eng. J. 9 (3) (1969) 3-12. ${ }_{644}$

[50] J. Koplik, T. Lasseter, One- and two-phase flow in network models of porous media, ${ }^{645}$ Chem. Eng. Cornrnun 26 (1984) 285-295.

[51] J. Stark, M. Manga, The motion of long bubbles in a network of tubes, Transport in ${ }_{647}$ Porous Media 40 (1) (2000) 201-218.

[52] M. I. J. van Dijke, K. S. Sorbie, Pore-scale network model for three-phase flow in mixed- ${ }_{649}$ wet porous media, Physical Review E 66 (2002) 046302.

[53] J. Bico, D. Quéré, Self-propelling slugs, Journal of Fluid Mechanics 467 (2002) 101-127. 651 
[54] V. Joekar-Niasar, S. Hassanizadeh, H. Dahle, Non-equilibrium effects in capillarity and ${ }_{652}$ interfacial area in two-phase flow: Dynamic pore-network modelling, Journal of Fluid ${ }_{653}$ Mechanics 655 (2010) 38-71.

[55] M. H. Jensen, A. Libchaber, P. Pelcé, G. Zocchi, Effect of gravity on the saffman-taylor ${ }_{655}$ meniscus: Theory and experiment, Physical Review A 35 (5) (1987) 2221.

[56] S. S. Leung, R. Gupta, D. F. Fletcher, B. S. Haynes, Gravitational effect on taylor flow ${ }_{657}$ in horizontal microchannels, Chemical Engineering Science 69 (1) (2012) 553 - $564 .{ }^{658}$ doi:http://dx.doi.org/10.1016/j.ces.2011.11.016. 659 URL http://www.sciencedirect.com/science/article/pii/S0009250911008153 660

[57] A. C. Payatakes, Dynamics of oil ganglia during immiscible displacement in water- ${ }_{661}$ ․ wet porous media, Annual Review Fluid Mechanics 14 (1982) 365-393. doi:10.1146/ ${ }^{662}$ annurev.fl.14.010182.002053.

[58] K. Yongman, J. Wan, T. J. Kneafsey, T. K. Tokunaga, Dewetting of silica surfaces ${ }_{664}$ upon reactions with supercritical $\mathrm{CO}_{2}$ and brine: pore-scale studies in micromodels, ${ }_{665}$ Environmental Science \& Technology 46 (2012) 4228-4235. doi:10.1021/es204096w. ${ }_{666}$ 


\section{Pore doublet model}

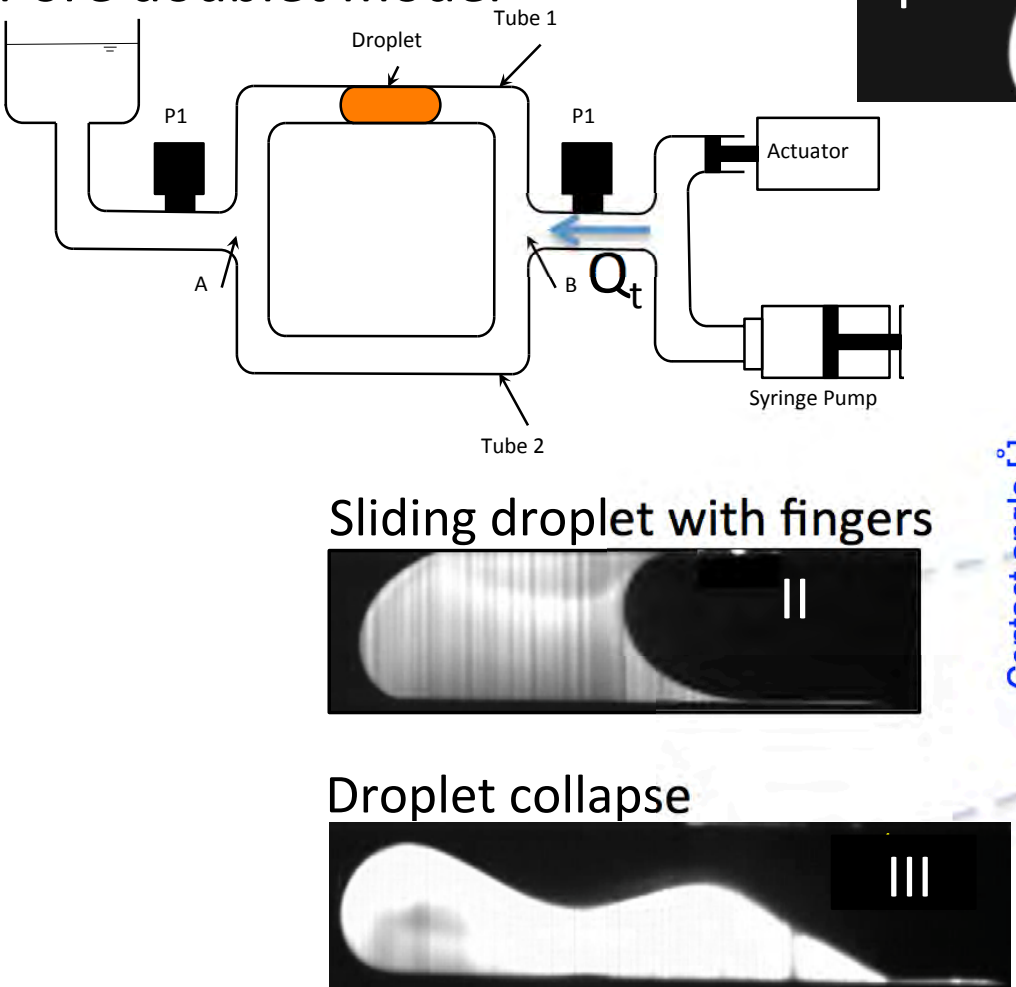

Droplet collapse

\section{III}

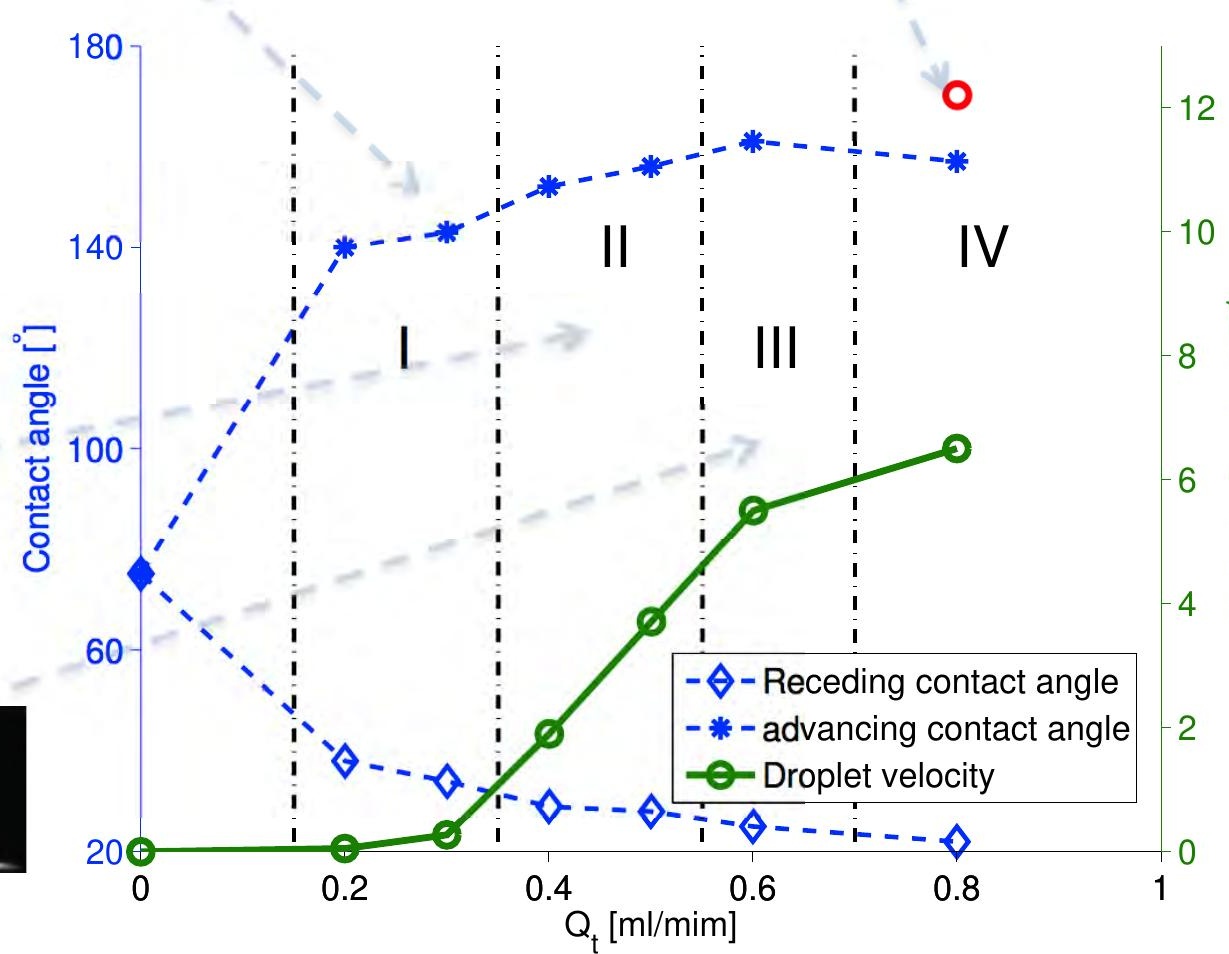

